\title{
AN ANALYSIS OF THE REPRESENTATIONS OF THE MAPPING CLASS GROUP OF A MULTI-GEON THREE-MANIFOLD
}

\author{
Rafael D. Sorkin ${ }^{a}$ \\ Departamento de Gravitación y Teoría de Campos \\ Instituto de Ciencias Nucleares, Universidad Nacional Autónoma de México \\ A. Postal 70-543, México D.F. 04510, México \\ and \\ Department of Physics, Syracuse University, Syracuse, N.Y. 13244-1130 \\ $\&$ \\ Sumati Surya ${ }^{b}$ \\ Department of Physics, Syracuse University, Syracuse, N.Y., 13244-1130 \\ ${ }^{a}$ email: sorkin@xochitl.nuclecu.unam.mx,sorkin@suhep.syr.edu \\ ${ }^{b}$ email: ssurya@suhep.syr.edu
}

\begin{abstract}
One knows that the distinct unitary irreducible representations (UIR's) of the mapping class group $G$ of a 3 -manifold $\mathcal{M}$ give rise to distinct quantum sectors (" $\theta$-sectors") in quantum theories of gravity based on a product spacetime of the form $\mathbb{R} \times \mathcal{M}$. In this paper, we study the UIR's of $G$ in an effort to understand the physical implications of these quantum sectors. The mapping class group of a 3-manifold which is the connected sum of $\mathbb{R}^{3}$ with a finite number of irreducible primes is a semi-direct product group. Following Mackey's theory of induced representations, we provide an analysis of the structure of the general finite dimensional UIR of such a group. In the picture of quantized primes as particles (topological geons), this general group-theoretic analysis enables one to draw several qualitative conclusions about the geons' behavior in different quantum sectors, without requiring an explicit knowledge of the UIR's corresponding to the individual primes. An important general result is that the classification of the UIR's of the so called particle subgroup (equivalently, the UIR's of $G$ in which the slide diffeomorphisms are represented trivially) is reduced to the problem of finding the UIR's of the internal diffeomorphism groups of the individual primes. Moreover, this reduction is entirely consistent with the geon picture, in which the UIR of the internal group of a prime determines the species of the corresponding quantum geon, and the remaining freedom in the overall UIR of $G$ expresses the possibility of choosing an arbitrary statistics (bose, fermi or para) for the geons of each species. For UIR's which represent the slides nontrivially, we do not provide a complete classification, but we find some new types of effects due to the slides, including quantum breaking of internal symmetry and of particle indistinguishability. In connection with the latter, a novel kind of statistics arises which is determined by representations of proper subgroups of the permutation group, rather than of the group as a whole. Finally, we observe that for a generic 3-manifold there will be an infinity of inequivalent UIR's and hence an infinity of "consistent" theories, when topology change is neglected.
\end{abstract}




\section{$\S 1$. Introduction}

In any quantum theory of gravity based on a spacetime topology of the product form* $\mathbb{R} \times \mathcal{M}$, one is led naturally to the existence of distinct quantum sectors labeled by the inequivalent unitary irreducible representations (UIR's) of the group $G$ of large diffeomorphisms, or "mapping class group", of the spatial 3-manifold $\mathcal{M}$ [1] [2] [3] [4] [5]. Indeed such inequivalent quantizations (or "theta-sectors") occur whenever the configuration space of a quantum system has a non-trivial first homotopy group. In the case of (asymptotically flat) gravity on $\mathbb{R} \times \mathcal{M}$, the configuration space $Q$ can be taken to be the space of all 3-geometries on $\mathcal{M}$, and $\pi_{1}(Q)$ is then isomorphic to the mapping class group (MCG) of $\mathcal{M}$. In this paper we analyze the UIR's of $G$ in a manner designed to bring out what the different quantum sectors represent physically.

In $\S 2$ we review the structure of the mapping class group $G$ of a three-manifold that is asymptotically $\mathbb{R}^{3}$. Such a manifold can always be expressed as a connected sum of $\mathbb{R}^{3}$ with prime manifolds. We will exclude handles from the primes, in which case $G$ is a semidirect product of three subgroups (defined relative to a given presentation of the connected sum): the "slides" of one prime through another, the "permutations" among identical primes, and the "internal diffeos" of the individual primes. In fact there is a triad of normal subgroups, (slides) $\subseteq$ (slides and internal diffeos $) \subseteq \mathrm{G}$, and associated with it, a semidirect product decomposition $G=$ (slides) $\ltimes$ (internals) $\ltimes$ (permutations), which is implicit in the literature. Our demonstration of this semidirect product form will use the concept of the "development" of a diffeomorphism on one hand, and on the other hand, the fact that the MCG is essentially identifiable with a subgroup of $\operatorname{Aut}\left(\pi_{1}(\mathcal{M})\right)$ whose structure, in turn, can be deciphered with the help of the Fuks-Rabinovitch presentation [6] of the automorphism group of a free product group. Part of this demonstration is contained in the Appendix.

In $\S 3$ we give a general analysis of the finite dimensional UIR's of semidirect product groups. Our exposition proceeds along the same lines as that of [7], but differs from it at certain points because for our purposes, one must consider projective representations of the normal subgroup without relaxing the condition that the overall UIR one is searching for be an ordinary (non-projective) representation of the full group. Full proofs for the structure theorems exposed in $\S 3$ may be found in [8] and [9]. In the subsequent sections

* In particular, canonically quantized gravity is included here, since it seems necessarily to be based on a spatial manifold of fixed topology. 
of the paper, we use the analysis of $\S 3$ to study the UIR's of the mapping class group of $\mathcal{M}$.

The results of this study lend credence to the interpretation of quantized primes as particles (topological geons). Indeed, when the slide diffeomorphisms are represented trivially, what remains is just a UIR of the so-called "particle-group" (internals) $\ltimes$ (permutations), which describes the symmetry of a collection of particles with internal structure. For this group, one is able to give explicitly the form of the most general finite dimensional UIR, and we do this in $\S 6$, using the structure theorems of $\S 3$. At the same time these structure theorems provide a terminology which (remarkably enough) allows one to characterize the corresponding quantum sectors entirely in the language of quantum particles and their properties, providing in this sense a complete physical interpretation for this class of UIR's. Within this interpretation, several general features emerge: the possibility of bosonic, fermionic and para-statistics; the loss of the spin-statistics correlation; and the quantum breaking of particle indistinguishability (i.e. the rendering distinct of classically indistinguishable objects).

For general UIR's of $G$, we obtain (in $\S \S 5$ and 6 ) only partial results because of our inability to categorize the UIR's of the slide subgroup. However these results (illustrated with the example of $\mathbb{R} P^{3}$ geons) already reveal several new effects associated with the slides, including the breaking of the internal symmetry of individual geons, and the rendering distinguishable of otherwise identical geons. With three or more geons the new effect emerges which is perhaps most striking: there are sectors in which the particle statistics is dictated by representations, not of the full permutation group, but by proper subgroups of it. In describing the effects in these terms, we are using the language appropriate to the structure theorems of $\S 3$; however there seem to be certain situations in which a slightly different language is more appropriate, and we explore this possibility briefly in $\S 6$.

As we will see, quantum gravity without topology change manifests in general an infinity of physically inequivalent quantum "sectors", an embarrassment of riches which seems at odds with the conception of quantum gravity as a fundamental theory. In the concluding section we argue that at a minimum, this indicates the necessity for incorporating topology change within quantum gravity. More realistically, it probably speaks to the existence of an underlying discrete microstructure, for which continuum quantum gravity can hope to provide only an effective low-energy description. 


\section{$\S 2$. The Mapping Class Group: its Role and Structure}

Let us first recall why the mapping class group (MCG) is relevant to quantum gravity. In an asymptotically flat product spacetime $\mathbb{R} \times \mathcal{M}$, where the spatial 3-manifold $\mathcal{M}$ is diffeomorphic outside of a compact region to $\mathbb{R}^{3}$, the natural configuration space $Q$ of general relativity is the space of all 3 -geometries on $\mathcal{M}$ which are asymptotically flat. Here a "3-geometry" is a diffeomorphism equivalence class of Riemannian metrics on $\mathcal{M}$; however not all diffeomorphisms should be considered "pure gauge" for this purpose. Rather, since physical observables like momentum and angular momentum can be expressed as surface integrals which generate diffeomorphisms at spatial infinity, only those diffeomorphisms that vanish at infinity should be considered gauge [1] [2] [5]. From a less formal point of view, we may say that a diffeomorphism which is non-trivial at infinity actually effects a change in the relation of the isolated system to its environment, and this is the physical reason why it would be wrong to treat it as gauge. Let $\operatorname{Diff}^{\infty}(\mathcal{M})$ be the group of all diffeomorphisms of $\mathcal{M}$ which vanish at $\infty$. Then if $\mathcal{R}$ is the space of all Riemannian metrics on $\mathcal{M}$ that are asymptotically flat, we have $Q=\mathcal{R} / \mathrm{Diff}^{\infty}$. Since the action of Diff $^{\infty}$ on $\mathcal{R}$ is free [1] [2] [5], $\mathcal{R}$ is in fact a principal fiber bundle with base space $Q$ and fiber isomorphic to Diff ${ }^{\infty}$.

Fundamentally, the fact that $Q$, and not $\mathcal{R}$ as such, is the natural configuration space of quantum gravity on $\mathbb{R} \times \mathcal{M}$, just expresses the postulate of "general covariance". However the formal meaning of this fact appears differently in different formulations of quantum gravity. From the starting point of canonical quantization in the Schrödinger representation, the so-called momentum constraints translate into invariance under Diff $0_{0}^{\infty}$, the subgroup of Diff ${ }^{\infty}$ which is connected to the identity (also called the subgroup of "small diffeomorphisms"). If implemented in the "Dirac" manner this invariance asserts that the "physical wave-functions" are in effect functions only over $\mathcal{R} / \operatorname{Diff}_{0}^{\infty}[10]$. The further step of "dividing out" by diffeomorphisms that are not in Diff ${ }^{\infty}$ must then be done "by hand". From a path integral starting point, in contrast, both the restricted and the full diffeomorphism invariances have clear geometrical meanings. Invariance under the spatial group $\operatorname{Diff}_{0}^{\infty}(\mathcal{M})$ now arises as a direct consequence of spacetime diffeomorphism invariance; while the extension of this invariance group to $\operatorname{Diff}{ }^{\infty}(\mathcal{M})$ occurs naturally as the result of summing over all possible ways of "attaching" the spacetime manifold to the "final hypersurface" $\mathcal{M}$, i.e., it arises as the consequence of a rudimentary type of topology change. 
The quotient group Diff ${ }^{\infty} /$ Diff $_{0}^{\infty}=: \pi_{0}\left(\right.$ Diff $\left.^{\infty}\right)$ is called the "mapping class group" (MCG) of $\mathcal{M}$; it is also often termed the "large diffeomorphism group". We shall denote it by $G$ and sometimes call it simply "the diffeo group". Given the fact that $\mathcal{R}$ is convex and hence homotopically trivial, the homotopy exact sequence for a principal fiber bundle leads to the isomorphism, $\pi_{1}(Q) \simeq \pi_{0}\left(\right.$ Diff $\left.^{\infty}\right)=G$.

Now, it is well known that the existence of a non-trivial fundamental group of the configuration space leads to inequivalent quantizations determined by the UIR's of that group. In the canonical framework, each inequivalent "sector" of Hilbert space is realized in terms of an $n$ dimensional vector-bundle over $Q$, where $n$ is the dimension of the corresponding UIR of $\pi_{1}(Q)$ and the state vectors or "wave functionals" are the cross sections of the vector bundle.

The role of the MCG emerges even more directly from a "path-integral" or "sumover-histories" starting point. In that framework the fundamental dynamical input is a rule attaching a quantum amplitude to each pair of truncated histories which "come together" at some "time" [11] [12]. Let us call such a pair a "Schwinger history" for short, and its underlying manifold a "Schwinger manifold". In the case of quantum gravity, each separate truncated history is a Lorentzian manifold with final boundary (and possibly initial boundary depending on the physical context), and the "coming together" means the identification or "sewing together" of the final boundaries. Now, as alluded to above, different ways of sewing are possible, related to each other by large diffeomorphisms of the final boundary. (This final boundary corresponds to the spacelike slice $\mathcal{M}$ of the canonical formulation). In general such a re-identification may or may not lead to a diffeomorphic Schwinger manifold, but it never will if we restrict ourselves to product spacetimes of the form $\mathbb{R} \times \mathcal{M}$, i.e. if we exclude topology change from the truncated histories (and if we limit ourselves to diffeomorphisms vanishing on any initial boundaries which may be present). In this case, the MCG of $\mathcal{M}$ acts freely and transitively (albeit non-canonically) on the set of Schwinger manifolds. Now, without disturbing the classical limit of the theory or the local physics, we can multiply the amplitude of each Schwinger history by a complex number depending only on the topology of the underlying manifold. Somewhat analogously to [13], one can then argue that consistency requires that these complex weights transform under some unitary representation of $G$, and that weights belonging to disjoint representations of $G$ "do not mix". The pure cases are then the UIR's of $G$, and we arrive again at the conclusion that each distinct UIR of the MCG yields an inequivalent version or "sector" of 
quantum gravity without topology change. Thus a study of the UIR's of the diffeo group is required to understand the possible inequivalent sectors of quantum gravity [1] [2] [5].

[ In the context of canonical quantization, two related questions might arise at this point, one concerning the role of the spacetime diffeomorphisms, as opposed to the purely spatial ones, and the second being the question why we should be dealing only with UIR's of the MCG, rather than general UIR's of the full invariance group Diff ${ }^{\infty}$. From the point of view of the wave-function, the spacetime diffeos do not appear as such, but, as is well known, their influence can be felt in a further set of restrictions on the wave-function, the "Hamiltonian constraints". (The precise content of these constraints depends on whether or not one adopts the so-called "unimodular" modification of general relativity [11]. If one does not adopt it, then there is, so to speak, one constraint for each point of the 3-manifold $\mathcal{M}$. If one does adopt it then one of these constraints disappears and a Schrödinger equation takes its place.) Unlike with the passage from Diff $0^{\infty}(\mathcal{M})$ to $\operatorname{Diff}^{\infty}(\mathcal{M})$, it does not seem possible to encode this infinity (or "infinity minus one" in the unimodular case) of constraints into a further contraction of the effective configuration space $Q$. On the other hand, the presence of this "extended gauge invariance" should probably be understood as the reason why it is only the quotient group $\pi_{0}$ (Diff ${ }^{\infty}$ ) (and not Diff ${ }^{\infty}$ itself), whose representations we need concern ourselves with. ]

For a generic 3-manifold the mapping class group is a cumbersome group to deal with since it is both infinite and discrete, and such groups tend to possess representations of types II and III, unless they are "almost abelian". (For example, the regular representation of an infinite discrete group $G$ is type II if the quotient of $G$ by the subgroup of all elements belonging to finite conjugacy classes is infinite [7].) But type II and III representations have the unsettling property of a non-unique decomposition into irreducibles, which would hamper any attempt to analyze the physical behavior of geons described by such representations solely in terms of UIR's of $G$. There is also the worry whether the infinite multiplicity of states associated with an infinite dimensional representation of $G$ can be physically acceptable. To avoid such problems, we will restrict ourselves in this paper to finite dimensional representations of $G$, for which the decomposition into UIR's is always unique. In addition the restriction to finite dimensionality will greatly simply the general analysis of $\S 3$ below.

The simplest example of the influence of the MCG is the existence of nontrivial $2 \pi$ rotations of the three manifold $\mathcal{M}$, implying the possibility of half-integer angular 
momentum in pure gravity [10] [14]. Since the $4 \pi$ rotation $i s$ trivial, one has only the two UIR's of the group $\mathbb{Z}_{2}$, and hence the choice between spinorial and tensorial sectors. Given the whole diffeo group of the three manifold then, it is natural to inquire whether other kinds of interesting behavior may be found, but before addressing this question, we must examine the MCG itself more closely.

\section{The structure of the mapping class group}

Let us consider first the case of oriented three-manifolds. Then [15], any manifold $\mathcal{M}$ that is diffeomorphic to $\mathbb{R}^{3}$ outside of a compact region can be decomposed uniquely as a connected sum of $\mathbb{R}^{3}$ with closed prime manifolds, which in this sense are the fundamental constituents of $\mathcal{M}$. We write this decomposition as $\mathcal{M}=\mathbb{R}^{3} \#\left(\#_{i} \mathcal{P}_{i}\right)$, where the connected sum operation \# is defined as follows. If $X$ and $Y$ are oriented three-manifolds, remove a 3-ball (or "disk") $\mathbf{D}^{3}$ from each of them, to get $X \backslash \mathbf{D}^{3}$ and $Y \backslash \mathbf{D}^{3}$, thus creating an $S^{2}$ boundary for each; then identify along these boundaries in an orientation-consistent manner to get the oriented manifold $X \# Y$. By a prime manifold $\mathcal{P}_{i}$ we mean, then, a closed three-manifold that cannot be further decomposed into a connected sum of other three manifolds. (The only possible further decomposition is trivial, and is a connected sum of the prime itself with a three sphere $S^{3}, \mathcal{P}_{i}=\mathcal{P}_{i} \# S^{3}$.) For some examples of prime manifolds see [16]. We will refer to the quantized primes as topological geons, or just "geons" for short.

For unoriented manifolds (including unorientable ones), the situation is similar but less simple to express, because of two kinds of ambiguity which can be present. First, the connected sum itself can be ambiguous, because there are two distinct ways to identify the bounding 2-spheres of the excised $\mathbf{D}^{3}$ 's, related to each other by a parity flip. When both $X$ and $Y$ are chiral, the two identifications will yield inequivalent (non-diffeomorphic) results for $X \# Y$. (A chiral manifold is an orientable manifold that admits no orientation-reversing diffeomorphism.) Thus, in forming an unoriented connected sum whose summands include $n$ chiral primes and no non-orientable primes, one has $2^{n-1}$ possible outcomes. ${ }^{*}$ In the geon language, one has a choice of whether each prime will occur as a certain geon or as the $C P$-conjugate geon (antiparticle). Notice that this ambiguity disappears if even one non-orientable prime is present. It would thus disappear entirely if we limited ourselves to manifolds which were either oriented or not orientable at all. The second kind of ambiguity

* In the asymptotically flat setting, we may regard $\mathbb{R}^{3}$ as a chiral prime. Then a sum of it with $n$ chiral (closed) primes can performed in $2^{n}$ different ways. 
is that the decomposition into primes is in general non-unique when handles are present. Specifically, if $\mathcal{M}$ is non-orientable then we have the isomorphism

$$
S^{2} \times S^{1} \# \mathcal{M} \simeq S^{2} \widetilde{\times} S^{1} \# \mathcal{M}
$$

where $S^{2} \times S^{1}$ is the "orientable handle", and $S^{2} \widetilde{\times} S^{1}$, its $\mathbb{Z}_{2}$-twisted analog, is the "nonorientable handle". Thus, the two types of handle are interchangeable summands in certain situations. However, we will be excluding handles from consideration altogether, and so the minor non-uniqueness they entail will be of no consequence for this paper.

We now exclude the handles by specializing to the case where $\mathcal{M}$ is a connected sum of irreducible primes, these being the closed three manifolds for which any embedded two-sphere $S^{2}$ can be shrunk to a point. The only orientable prime three-manifold which is not irreducible is the $S^{2} \times S^{1}$ handle. (Sometimes called "wormhole", this is probably the best known - if least generic — of all primes). The diffeomorphism group of a manifold containing such handles exhibits some peculiarly nonlocal behavior that interferes with a particle-like interpretation of handle primes [17] [18] [19]. Possibly, one could attain a consistent particle interpretation by treating the ends of a handle as separate entities in certain situations (i.e. in certain regions of state space), but we will not explore that possibility herein. Instead, we will simply limit ourselves to manifolds $\mathcal{M}$ not containing summands $S^{2} \times S^{1}$ or $S^{2} \widetilde{\times} S^{1}$. Notice that with the exclusion of handles, the prime decomposition of $\mathcal{M}$ becomes unique, even in the unoriented case.

Having excluded handles, we now also limit ourselves to orientable manifolds $\mathcal{M}$. Unlike with the handles, we know of no impediment to extending our analysis to the non-orientable case. On the contrary, there are interesting new features (like interconversion between particles and anti-particles) which seem to fit well into our scheme. Rather we exclude nonorientable primes only because, at several points, we use theorems whose nonorientable extensions we have not been able to find in the literature. However, we believe these extensions exist (mutatis mutandis), and we will at various points comment on the nonorientable case, always with the proviso that what we say depends on the continued validity of certain theorems known to obtain in the orientable case.

Let us fix a concrete representation of $\mathcal{M}$ as a connected sum of primes (a "presentation"). It is well documented in the mathematics literature [17] 20] [21], that with respect to such a presentation, the $\mathrm{MCG}$ of $\mathcal{M}$ is generated by the following elements, a description of which can be found in [2] 
(a) internal diffeos of the individual primes

(b) exchanges of identical primes

(c) slides of primes through other primes along generators of $\pi_{1}(\mathcal{M})$.

Let us examine the structure of the MCG more closely, in the case in which $\mathcal{M}$ is a connected sum of $N$ identical primes $\mathcal{P}_{i}$. (The general case is more complicated only in notation.) For our subsequent analysis the important circumstance will be that $G$ is the semidirect product of the three subgroups generated by the three types of diffeomorphisms just enumerated.* We will call these respectively the subgroup $G_{i n t}$ of internal diffeomorphisms, the permutation subgroup $S_{N}$, and the slide subgroup $\mathcal{S}$. In the next few paragraphs we discuss the structure of these three subgroups, and we describe the manner in which $G$ is their semidirect product. Some of the relevant facts we will state without proof, giving demonstrations only insofar as they seem helpful for arriving at a physical interpretation of the various elements of $G$ and its UIR's. In the Appendix, we describe how to make the proofs complete (assuming the Poincaré conjecture to be true.)

The subgroup of internal diffeomorphisms (the subgroup generated by the diffeomorphisms of type (a)) is the direct product

$$
G_{i n t}=G_{1} \times G_{2} \times \cdots \times G_{N}
$$

where $G_{i}$ is the MCG of $\mathbb{R} \# \mathcal{P}_{i}$, and the $G_{i}$ are mutually isomorphic, because all the primes are identical. The product is direct since the support of each $G_{i}$ is by definition restricted to the region inside the $i^{\text {th }}$ separating sphere (which as its name indicates, separates the $i^{\text {th }}$ prime from the rest of $\mathcal{M}$ ), implying that the internal diffeos corresponding to different primes do not mix (see the Appendix).

The permutation subgroup (the subgroup of $G$ generated by diffeomorphisms of type (b)) consists of the $N$ ! diffeomorphisms that permute the $N$ identical primes $\mathcal{P}_{i}$ amongst themselves. (When $\mathcal{P}$ is nonorientable, we assume for purposes of defining the exchanges that the presentation of $\mathcal{M}$ has been chosen so that all the $\mathcal{P}_{i}$ are diffeomorphic via translation through the ambient $\mathbb{R}^{3}$.) Thus (see the Appendix) its structure is just that of the permutation group on $N$ elements, and we have given it the same name, $S_{N}$.

The slide subgroup $\mathcal{S}$ (the subgroup of $G$ generated by diffeomorphisms of type (c)) is the free product

$$
*_{i} *_{j} \mathrm{~S}_{i j} \quad(i, j=1 \cdots N, i \neq j)
$$

\footnotetext{
* It is this semidirect product decomposition that fails when handles are present.
} 
modulo certain geometrically evident commutation relations, where $\mathrm{S}_{i j} \simeq \pi_{1}\left(\mathcal{P}_{j}\right)$ comprises the slides of the $i^{\text {th }}$ prime through the $j^{\text {th }}$ prime (that is, an element of $\mathrm{S}_{i j}$ slides $\mathcal{P}_{i}$ along a loop belonging to $\left.\pi_{1}\left(\mathcal{P}_{j}\right)\right)$. When only two primes are present $\mathcal{S}$ is literally the free product of $\mathrm{S}_{12}$ with $\mathrm{S}_{21}$. However when three or more primes are present certain commutation relations among the generators of distinct subgroups must be imposed, as described in [6] [21]. For example for $N=4, \mathrm{~S}_{12}$ obviously commutes with $\mathrm{S}_{34}$. A complete set of these relations is as follows:

$$
\mathrm{s}_{i j} \downarrow \mathrm{s}_{k l}, \quad \mathrm{~s}_{i j} \downarrow \mathrm{s}_{k j}, \quad \mathrm{~s}_{i k} \mathrm{~s}_{j k} \downarrow \mathrm{s}_{i j},
$$

where $\{i, j, k, l\}$ label distinct primes, $\mathrm{s}_{i j}$ is any generator of $\mathrm{S}_{i j}$, and the symbol $\downarrow$ denotes commutability:

$$
A \emptyset B \equiv A B=B A .
$$

Generators for the groups $\mathrm{S}_{i j}$ can be chosen as follows. Let $\gamma_{i}^{\phi}$ be generators of $\pi_{1}\left(\mathcal{P}_{i}\right)$ where $\phi$ runs from 1 to $n$. Clearly there will be $n \times N$ such $\gamma_{i}^{\phi}$ in all. Now for each such generator passing through the $i^{t h}$ prime, there is a slide of any one of the other primes along it. Let $s_{i}^{\phi, j}$ denote the slide of the $i^{\text {th }}$ prime along the $\phi^{t h}$ generator of $\pi_{1}$ of the $j^{\text {th }}$ prime. There will be $n N(N-1)$ such generators in all, since a prime cannot slide through itself. (In the non-orientable case, some of these generators can fail to exist, when more than one type of prime is present. Thus, if one attempts to slide a chiral prime $\mathcal{P}_{i}$ along an orientation reversing loop $\gamma \subseteq \mathcal{M}$, one fails because $\mathcal{P}_{i}$ returns as the mirror image of itself, which (in the language used below) precludes the would-be "development loop" from closing. Hence, the group defined by the generators and relations just described contains spurious elements, and the actual $\mathcal{S}$ is a subgroup of it, namely the subgroup of elements which contain an even number of orientation reversing generators $s_{i}^{j}$ for each chiral prime $\left.\mathcal{P}_{i}.\right)$

A second subtlety concerning the slides involves spinorial primes (those whose $2 \pi$ rotation is nontrivial). Above we wrote that the generators (a)-(c) were defined relative to a presentation of $\mathcal{M}$ as a connected sum. We note here that such a presentation involves more than just a choice of separating $S^{2}$ 's and of identifications among the identical primes. It requires as well a distinguished notion of "parallel transport" of the primes to enable one to disentangle the "pure slides" from "slides mixed with $2 \pi$-rotations of the sliding primes". For a fuller discussion of this point see [5]. (In the non-orientable case one can transport chiral primes with respect to the orientable double cover of $\mathcal{M}$. We believe a 
similar technique should work also for the transport of nonorientable primes and non-chiral orientable primes.)

Given the slides, the internal diffeos and the permutations, one can build up the entire mapping class group $G$ as the semidirect product

$$
G=\mathcal{S} \ltimes G_{\text {int }} \ltimes S_{N}
$$

(By writing $G=A \ltimes B \ltimes C$ we mean the following. Every $g \in G$ is uniquely of the form $g=a b c$ with $a \in A, b \in B, c \in C$; and for all such $a, b, c$, we have $b a b^{-1} \in A, c a c^{-1} \in A$ and $c b c^{-1} \in B$. This is equivalent to $G=A \ltimes(B \ltimes C)$ and implies $G=(A \ltimes B) \ltimes C$.) The proof of this semidirect product structure is described in the Appendix, relying heavily on the device of replacing the study of $G$ as such by the study of its action on $\pi_{1}(\mathcal{M})$. Here we limit ourselves to a partial treatment intended to be useful in gaining an intuitive understanding of the structure of $G$.

First, let us show that the slide subgroup $\mathcal{S}$ is normal in $G$, as required by the decomposition (2). Consider the slide $s$ of a prime $\mathcal{P}_{i}$ along a generator of $\pi_{1}(\mathcal{M})$ that passes through the prime $\mathcal{P}_{j}$, say. This slide will commute with all permutations of the primes except those involving $\mathcal{P}_{i}$ and $\mathcal{P}_{j}$, and with all internal diffeos but those of $\mathcal{P}_{j}$. When conjugated by permutations involving $\mathcal{P}_{i}$ or $\mathcal{P}_{j}, s$ becomes another slide, now between the permuted primes. Thus, if we can show that conjugation of $s$ by an internal diffeo of $\mathcal{P}_{j}$ also yields another slide, then we can state that the slides form a normal subgroup in the diffeo group. We proceed to do this using the idea of the development of a diffeomorphism.

A diffeo is said to be developed in the following sense [2]. If you start off with the manifold $\mathcal{M}$ and "implement the diffeomorphism in a continuous manner", then you obtain a sequence of manifolds that are diffeomorphic to $\mathcal{M}$ but different, except when the diffeo is completed, in which case one is back to the same manifold as before. That is, the development is a loop of manifolds that are diffeomorphic to each other, with the base point being $\mathcal{M}$, and such that in going around the loop, one has in effect performed the above diffeo. For example, the development of a diffeomorphism exchanging two $T^{3}$ primes is illustrated in figure 1. 


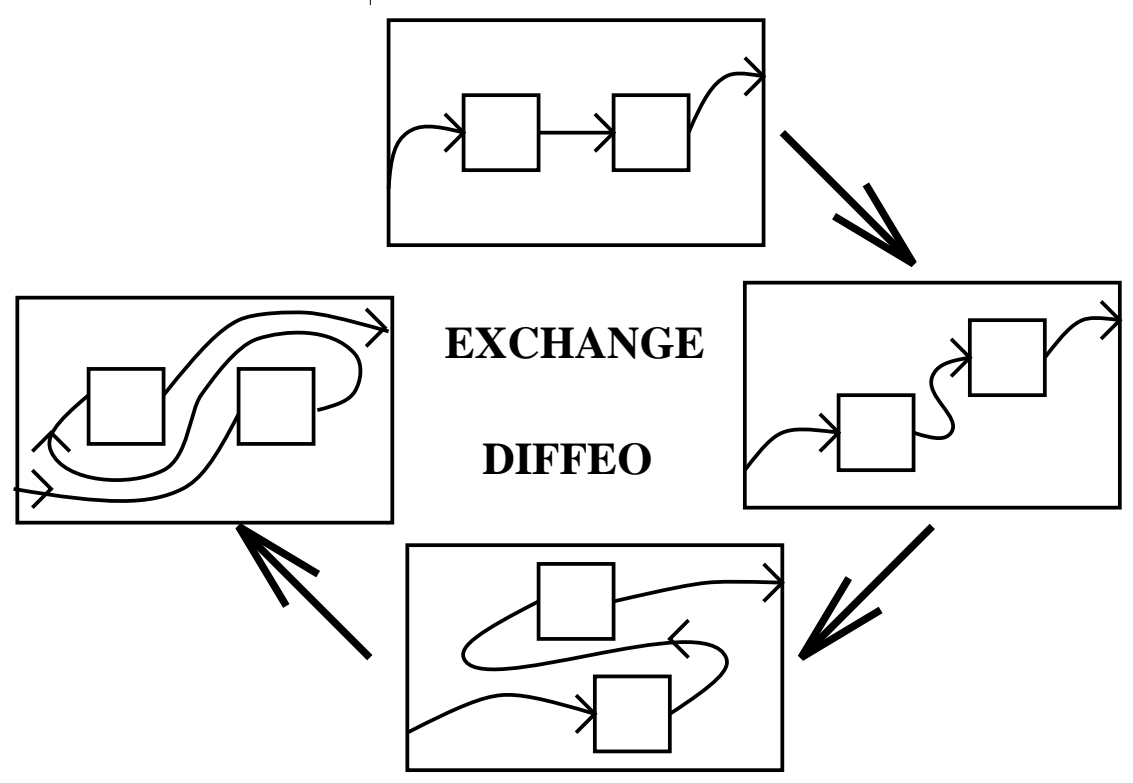

Figure 1. Development of the exchange diffeomorphism for two $T^{3}$ primes. The line with arrows shows the effect of the diffeomorphism on a curve whose end points are fixed at infinity and which loops through the two primes non-trivially.

The diffeo we are interested in is $g s g^{-1}$ where $g$ is the internal diffeo and $s$ is the slide diffeo. So we develop $g^{-1}$ first, then $s$ and then $g$. This gives us a single loop of manifolds, as illustrated in figure 2. The aim now is to show that this loop is in fact deformable to one that develops just a slide. To do so, we can proceed as follows. Instead of completing all of $g^{-1}$ we leave a little bit of this diffeomorphism undone (hence the manifold thus obtained is different from the one we started out with). The slide is now developed by taking it along the "same" generator as before. The remaining bit of $g^{-1}$ is then completed and finally $g$ is developed exactly as before. The result is a slightly different loop of manifolds from originally. Now we keep decreasing the amount of $g^{-1}$ that is developed before the slide is executed so that we obtain a whole sequence of loops parameterized continuously by some $\epsilon \in[0,1]$ which measures the "amount" of $g^{-1}$, " $\epsilon g^{-1}$ ", that has been left undone before the slide is executed. The final loop in this sequence is one for which none of $g^{-1}$ is done before the slide, and the slide is along a new generator obtained by continuity from the original one. Of course, developing $g^{-1}$ and $g$ consecutively gives the identity, and thus this last loop involves only a slide along a generator that is different from the one for the original slide. 


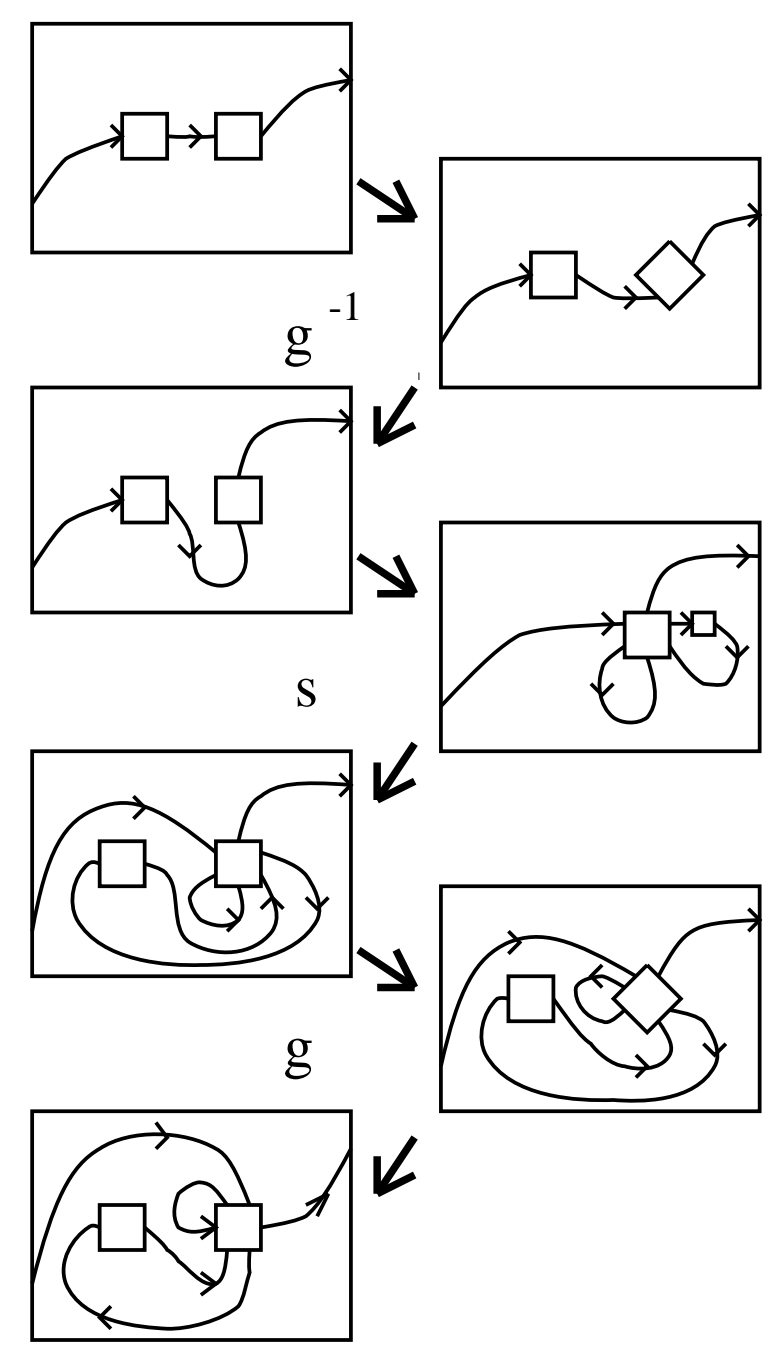

Figure 2. Development of the diffeomorphism $g_{s g^{-1}}$ for two $T^{3}$ primes. Here $g$ is an "internal rotation" by $\pi / 2$, and $s$ is a slide of the first prime through the second. Panels 1-3 develop $g^{-1}, 3-5$ develop $s$, and 5-7 develop $g$.

A generic loop in our sequence of developments can be described in the following way. Perform $\epsilon g^{-1}$ first, then slide $\mathcal{P}_{i}$ along the same generator of $\mathcal{P}_{j}$ that it goes through when $\epsilon=1$. Finish the development by doing $(1-\epsilon) g^{-1}$ and then $g$. Since these loops are all deformable into each other, the diffeomorphisms they develop are isotopic to each other: they yield the same element of $G=\pi_{0}\left(\right.$ Diff $\left.^{\infty}\right)$. Thus we have shown that conjugation of a slide diffeo by an internal diffeo of the prime slid through is a slide along another generator of the same prime. Hence the slide subgroup is normal in the full mapping class group $G$.

In the same way, it is clear that for any $g_{i n t} \in G_{i n t}$ and any $p \in S_{N}, p g_{i n t} p^{-1}$ is again an internal diffeo. For example, imagine that $p$ interchanges the $i^{\text {th }}$ prime with the 
$j^{t h}$ one and that $g$ is an internal diffeomorphism of the $i^{t h}$ prime. Then conjugation of $g$ with $p$ gives the corresponding internal diffeo of the $j^{\text {th }}$ prime. Thus we have demonstrated everything needed for (2) but the uniqueness of the decomposition. This is done in the Appendix.

Finally, consider the quotient group $G / \mathcal{S}$, which by (2) may be identified with the subgroup $\widetilde{G} \subseteq G$ generated by the elements of $S_{N}$ and $G_{i n t}$ (i.e., $\widetilde{G}=G_{i n t} S_{N}$ ). This group has been dubbed the particle group, since its elements have natural interpretations as symmetry operations on particles possessing internal structure. By (2), it also is a semidirect product, and this fact will be the key, both to our analysis of its representations and to their physical interpretation.

The important structural result of this section is that $G$ can be expressed as a semidirect product of three subgroups with clear physical meanings. Since we are interested in understanding the finite dimensional UIR's of $G$, it is therefore important to understand in general the structure of the UIR's of semidirect product groups. To that end, we provide the analysis of the next section.

\section{$\S 3$. The Structure of Representations of Semidirect Product Groups}

Consider a group $G$ with normal subgroup $N$ and quotient group $K=G / N$. In the language of exact sequences we express this situation as

$$
1 \rightarrow N \rightarrow G \rightarrow K \rightarrow 1
$$

We will assume in addition that this sequence splits, meaning that its projectionhomomorphism, $\pi: G \rightarrow K$ possesses a "section" or right inverse, i.e., that there exists a homomorphism $j: K \rightarrow G$ such that $\pi j=1_{K}$. In less poetic language what we are saying is that $G$ can be realized as a semi-direct product, $G=N \ltimes K$, which we do by using $j$ to construe $K$ as a subgroup of $G$ complementary to $N$. The precise realization of $K$ as a subgroup of $G$ depends on the splitting homomorphism $j$, but we will choose one such $j$ and never change it. We then have the situation:

$$
N \subseteq G, K \subseteq G, N \cap K=\{e\}, G=N K
$$

where $e$ is the identity element of $G$. (The ambiguity in $j$ is not an irrelevant technicality for our application to geons: it corresponds to the ambiguity in the presentation of $\mathcal{M}$ as a 
connected sum, and is linked intimately to the subtle distinction between concrete primes and the corresponding physical particles (geons). See [2] and [3].)

We aim to understand the general finite dimensional unitary irreducible representation (UIR) of G, by analyzing it in terms of representations of $N$ and representations of (the appropriate subgroups of) $K$. In fact one can begin with an arbitrary UIR $R: G \rightarrow A u t(\mathcal{H})$ and analyze it into certain structures involving $N$ and $K$. Conversely one can show that, beginning with these structures one can build up a representation of $G$ in a unique manner. These two inverse processes are described fully in [9]; here we merely summarize their main features. References [8] and [7] may also be consulted for much of this material, including an explanation of any terminology not defined here.

In what follows we use the symbol $\simeq$ to denote isomorphism of groups, vector spaces or group representations as the case may be. Similarly $\otimes$ will stand for several types of tensor product: of vectors, operators or representations. In the case of representations we make the following convention. If $R_{1}$ and $R_{2}$ are representations of different groups, $G_{1}$ and $G_{2}$, then $R_{1} \otimes R_{2}$ will denote the tensor-product representation of the direct product group $G_{1} \times G_{2}$. However, when $G_{1}=G_{2}=G$, then $R_{1} \otimes R_{2}$ will denote not a representation of $G \times G$, but the tensor product representation of $G$ itself (the latter or "inner product" representation of $G$ being just the restriction of the former or "outer product" representation of $G \times G$ to the diagonal subgroup within $G \times G$ ). In each case the context should make clear which product is intended.

The "Little Group" and Associated Structures

Before proceeding to the analysis of $R$, we must expose some structures and relations which are available independently of reference to any particular UIR of $G$. To begin with, let $\widehat{N}$ be the set of (finite dimensional) UIR's of $N$, up to equivalence; i.e., an element of $\widehat{N}$ is an equivalence class of concrete representations $\Gamma$ of $N$, where $\Gamma_{1} \simeq \Gamma_{2} \Longleftrightarrow \exists$ some unitary $S$, such that $\forall n \in N$,

$$
S \Gamma_{1}(n)=\Gamma_{2}(n) S
$$

Because $N$ is normal in $G, K$ acts naturally on $\widehat{N}$, the action of $k \in K$ on the UIR $\Gamma$ of $N$ being to produce the UIR $\Gamma^{\prime}$ defined by

$$
\Gamma^{\prime}(n)=\Gamma\left(k n k^{-1}\right), \forall n \in N
$$


We will express this relationship by writing $\Gamma^{\prime}=\Gamma k$, placing $k$ on the right since our definition of $\Gamma^{\prime}$ yields a right action in the sense that $\left(\Gamma k_{1}\right) k_{2}=\Gamma\left(k_{1} k_{2}\right)$. Since $\Gamma_{1} \simeq \Gamma_{2} \Rightarrow$ $\Gamma_{1} k \simeq \Gamma_{2} k$, we acquire an action $[\Gamma] \mapsto[\Gamma] k$ of $K$ on $\widehat{N}$ (where $[\Gamma]$ means the equivalence class in $\widehat{N}$ of $\Gamma$ ).

Now, let $\mathcal{O} \subseteq \widehat{N}$ be an orbit of the $K$-action just defined. If we choose a fiducial element $\left[\Gamma_{0}\right] \in \mathcal{O}$, then we can define the associated "little group" $K_{0}$ to be the "stability subgroup" of $K$ with respect to $\left[\Gamma_{0}\right]$ :

$$
K_{0}=\left\{k \in K \mid\left[\Gamma_{0}\right] k=\left[\Gamma_{0}\right]\right\}
$$

In the following we will assume that such a fiducial element has been chosen, once and for all, in every orbit $\mathcal{O}$.

If, further, we choose a fiducial representative, $\Gamma_{0} \in\left[\Gamma_{0}\right]$, then we acquire a projective unitary representation of $K_{0}$ as follows. By definition, $\Gamma_{0} k \simeq \Gamma_{0}$ for $k \in K_{0}$. But this means

$$
\Gamma_{0}\left(k n k^{-1}\right)=S(k) \Gamma_{0}(n) S(k)^{-1}
$$

for some unitary operator $S(k)$ acting in the carrier space of $\Gamma_{0}$. Since $n$ in (8) is arbitrary, $S(k)$ is thereby determined uniquely up to phase. Compatibility with group multiplication in $K$ then implies that

$$
S\left(k_{1}\right) S\left(k_{2}\right)=\sigma\left(k_{1}, k_{2}\right) S\left(k_{1} k_{2}\right)
$$

where $\sigma: K_{0} \times K_{0} \rightarrow U(1)$ is a projective multiplier for $K_{0}$. We remind the reader that a fixed, concrete $\Gamma_{0}$ has been chosen for every orbit, and we hereby choose also a fixed set of phases for the $S(k)$. (Of course we will choose these phases so that $\sigma \equiv 1$ when that is possible).

To summarize: Given the split exact sequence $1 \rightarrow N \rightarrow G \rightarrow K \rightarrow 1$ (together with choices of certain fiducial elements where necessary) we acquire automatically:

- an action of $K$ on the UIR's of $N$; and for each orbit $\mathcal{O}$ of this action in $\widehat{N}$ :

- the corresponding stability group (or "little group") $K_{0} \subseteq K$;

- a projective multiplier $\sigma$ for $K_{0}$; and

- a projective representation $S$ of $K_{0}$ with multiplier $\sigma$ (with $S$ acting in the carrier space $\mathcal{H}_{0}$ of the fiducial representation $\Gamma_{0}$ chosen for the orbit $\mathcal{O}$ ).

To acquire these structures we have had to choose certain fiducial elements, and one may be curious how an altered choice would affect the above acquisition list. The 
choices made comprise: a choice of splitting map $j$; and for each orbit $\mathcal{O}$ : a choice of UIR $\Gamma_{0}$ with $\left[\Gamma_{0}\right] \in \mathcal{O}$ plus a choice of phase for each operator $S(k)$. Changing the splitting map $j$ alters the subgroup of $G$ which represents $K=G / N$, but it does not affect the action of $G / N$ on $\widehat{N}$. It also need not affect the projective multiplier $\sigma$, although it does necessarily modify (in a simple manner) the operators $S(k)$. Changing the fiducial element $\left[\Gamma_{0}\right] \in \mathcal{O}$ changes $K_{0}$ by an inner automorphism of $K$, and both $S$ and $\sigma$ can be given the new values induced by this inner automorphism; in that sense they remain unaffected. Changing which $\Gamma_{0}$ represents the fiducial element of $\mathcal{O}$ need have no effect other than a similarity transformation on the operators $S(k)$. Finally, changing the phases of the $S(k)$ modifies only $\sigma$, taking it by definition to another member of the same projective equivalence class $[\sigma]$ (i.e. another cocycle representing the same element of the group cohomology of $\left.K_{0}\right)$.

Analysis of a UIR of $\mathrm{G}$

Let $R: G \rightarrow A u t(\mathcal{H})$ be a finite dimensional unitary irreducible representation (UIR) of $G=N \ltimes K$ acting in the Hilbert space $\mathcal{H}$, and let $\widetilde{R}=R \mid N$ be its restriction to the normal subgroup $N$ (i.e. $\widetilde{R}: N \rightarrow A u t(\mathcal{H})$ via $\widetilde{R}(n)=R(n)$ ). The analysis of $R$ then runs as follows.

First, $\widetilde{R}$ must (up to a similarity transformation) take the form

$$
\widetilde{R}:=R \mid N=\left(\bigoplus_{[\Gamma] \in \mathcal{O}} \Gamma\right) \otimes \mathbf{1}_{\beta}
$$

where $\mathcal{O} \subseteq \widehat{N}$ is some definite $K$-orbit (determined by $R$ ) and $\mathbf{1}_{\beta}$ denotes the trivial representation of $N$ in $\mathbb{C}^{\beta}$. Here, the direct sum notation denotes a sum of UIR's taken over the orbit $\mathcal{O}$. That is, we select from each equivalence class $\alpha_{i} \in \mathcal{O}(i=0, \cdots,|\mathcal{O}|-1)$ a representative representation $\Gamma_{i} \in \alpha_{i}$, and we form the direct sum representation $\bigoplus_{i=0}^{|\mathcal{O}|-1} \Gamma_{i}$. For $i=0$ we will make the convention that $\alpha_{0}$ is the fiducial equivalence class in $\mathcal{O}$, and we of course use the previously selected fiducial $\Gamma_{0} \in \alpha_{0}$ as $\Gamma_{i}$ when $i=0$.

Now let $K_{0}$ be the little group of $\mathcal{O}$, as always, and let $S: K_{0} \rightarrow \operatorname{Aut}\left(\mathcal{H}_{0}\right)$ be the corresponding projective representation of $K_{0}$ with multiplier $\sigma$, as described above. From (10), one component of $\widetilde{R}$ is the representation $\Gamma_{0} \otimes \mathbf{1}_{\beta}$ acting in the subspace $\mathcal{H}_{0} \otimes \mathbb{C}^{\beta}$. Then the restriction of the full representation $R$ to $K_{0}$ induces in this subspace a representation of the little group $K_{0}$ which has the form,

$$
S \otimes T
$$


where $T$ is some projective unitary irreducible representation (PUIR) of $K_{0}$ with multiplier $\tau=1 / \sigma$. The representation $T$ and the orbit $\mathcal{O}$ together contain enough information to identify uniquely the representation $R$ from which they came.

Note here that $T: K_{0} \rightarrow \operatorname{Aut}\left(\mathbb{C}^{\beta}\right)$ is not merely a projective equivalence class, but is a concrete collection of operators $T(k)$. Unfortunately, it too is not uniquely determined, but depends on the presentation of $\mathcal{H}_{0} \otimes \mathbb{C}^{\beta}$ as a tensor product. However a change of this presentation will only induce a similarity transformation, $T(k) \mapsto U T(k) U^{-1}$. Notice also, that a change to the phases chosen for the $S(k)$ would not alter the projective equivalence classes of $T$ and $\tau$. In fact a phase change which just multiplied $S(k)$ by a unitary character $\chi(k)$ of $K_{0}$ would not alter $\tau$ at all (since it would not alter $\sigma$ ).

To summarize: We have "analyzed" $R$ into a pair of structures: an orbit $\mathcal{O} \subseteq \widehat{N}$, and a PUIR $T$ of the corresponding little group $K_{0}$ with multiplier $1 / \sigma$ ( $\sigma$ being the projective multiplier associated to $\mathcal{O}$ as described as above). $T$ is determined by $R$ up to a similarity transformation, $T \rightarrow U T U^{-1}$.

Synthesis of a UIR of G

Now let us retrace our steps. Given any finite $K$-orbit $\mathcal{O} \subseteq \widehat{N}$ and any finite dimensional PUIR $T: K_{0} \rightarrow \operatorname{Aut}\left(\mathcal{H}_{T}\right)$ of the corresponding little group with projective multiplier $\tau=1 / \sigma$, we can construct a finite dimensional UIR $R$ of $G$. In this process we begin by representing $N$ and $K_{0}$ jointly in a certain "core" Hilbert space, and then we build up the rest of $\mathcal{H}$ by acting on the core with elements of $K / K_{0}$.

Specifically, let $\mathcal{H}_{0}$ be the carrier space of $\Gamma_{0}$ and let $\mathcal{H}_{T}$ be that of $T$ (so $\mathcal{H}_{T} \simeq \mathbb{C}^{\beta}$ where $\beta=\operatorname{dim} T)$. Then our "core", is the tensor product space,

$$
\mathcal{H}_{0} \otimes \mathcal{H}_{T}
$$

and our representation of $N K_{0}$ is that for which $n \in N$ acts as $\Gamma_{0}(n) \otimes \mathbf{1}$ and $k \in K_{0}$ acts as $S(k) \otimes T(k)$.

So far we have a UIR of $N K_{0} \subseteq G$ in $\mathcal{H}_{0} \otimes \mathcal{H}_{T}$. The full carrier space (or "substrate") $\mathcal{H}$ of $R$ is then built up in a unique manner as the direct sum of images of $\mathcal{H}_{0} \otimes \mathcal{H}_{T}$ by the operators $R(k)$ representing elements of $K$ which are not in $K_{0}$, there being one such image for each coset $K_{0} k \subseteq K$. Specifically, we can proceed by choosing a representative element $k$ from each coset and using the group multiplication rules of $G$ to determine how a general $R(g)$ acts on the resulting $\mathcal{H}$. This process of building up $R$ from our core UIR is called "induction", and there are in fact several versions of the 
construction available in the mathematics literature. The important point is that $R$ is determined uniquely (up to equivalence) once the "core representation" is given.

To summarize: Given a $K$-orbit $\mathcal{O} \subseteq \widehat{N}$, let $\sigma$ be the associated projective multiplier and $K_{0}$ be the associated little group. Assume that $\operatorname{card}(\mathcal{O})<\infty$. Then given in addition a PUIR $T$ of $K_{0}$ with multiplier $\tau=1 / \sigma$, we acquire a unique $[R] \in \widehat{G}(\widehat{G}$ being the set of equivalence classes of finite dimensional UIR's of $G$ ). Moreover, any two $T$ 's in the same similarity class yield equivalent $R$ 's.

What determines a UIR of G?

The procedures of analysis and synthesis just described are inverses of each other. Hence, taking them together, we have a bijective correspondence,

$$
(\mathcal{O}, \operatorname{cls}(T)) \leftrightarrow[R]
$$

where $\operatorname{cls}(T)$ is a similarity class of PUIR's $T: K_{0} \rightarrow \operatorname{Aut}\left(\mathcal{H}_{T}\right)$ and $[R]$ is an equivalence class of UIR's of $G$. That is: finding all finite dimensional UIR's of $G$ is equivalent to finding all pairs $(\mathcal{O}, T)$, where $\mathcal{O}$ is $K$-orbit in $N$ with finite cardinality, and $T$ is a collection of operators $T(k), k \in K_{0}$, which act irreducibly in a finite dimensional vector space and obey

$$
T\left(k_{1}\right) T\left(k_{2}\right)=\left(\sigma\left(k_{1}, k_{2}\right)\right)^{-1} T\left(k_{1} k_{2}\right),
$$

$\sigma$ being the projective multiplier associated with the orbit $\mathcal{O}$. For a given $\mathcal{O}$, two such $T$ 's determine the same equivalence class $[R]$ iff they are related by a similarity transformation,

$$
T^{\prime}(k)=U T(k) U^{-1} \quad\left(U^{*}=U^{-1}\right)
$$

We should stress here that a similarity class of PUIR's is not the same thing as a projective equivalence class. For the PUIR's $T$ and $T^{\prime}$ to be similar, the equality (15) must hold with a fixed $U$ for all $k$. For projective equivalence an arbitrary $k$-dependent phasefactor is allowed to intervene. For projective representations, the most natural notion of equivalence is projective equivalence. However, a projective equivalence class $[T]$ will in general determine a whole family of UIR's $[R]$, the elements of the family being related by the action of the character group of $K_{0}$ which takes $T \mapsto \chi T, \chi$ being the character. This action leaves the multiplier $\tau$ alone, but it modifies $[R]$, unless $\chi T$ happens to be similar to $T$. Thus we can also think of the UIR's of $G$ as being parameterized (for fixed $\mathcal{O}$ ) by a projective equivalence class $[T]$ of PUIR's $K_{0}$ together with an element of the quotient of 
the (unitary) character group of $K_{0}$ by the subgroup of characters that induce similarity transformations on $T$ :

$$
(\mathcal{O},[T],[\chi]) \longleftrightarrow[R]
$$

We should mention here that none of these subtleties involving projective representations will figure in the part of our subsequent analysis dealing with the "particle group", as in that case, the projective multiplier $\sigma$ will turn out to be trivial. However we know of no reason why nontrivial $\sigma$ would not arise in the analysis of the full MCG (with the slides playing the part of the normal subgroup $N$, and the particle subgroup playing the role of the quotient group $G / N=K)$.

In that situation, the full machinery of the present section would be needed. Moreover, one would then need to understand certain projective representations of the little group $K_{0} \subseteq K$, whence (as $K=\widetilde{G}$ would itself be a semi-direct product) an analysis of the projective representations of semi-direct product groups would likely be pertinent. A treatment of that problem can be found in [7], which indeed furnished the pattern on which much of the above is based. However, in [7] the subtleties involving the character group of $K_{0}$ do not appear, because that reference aims only to classify the PUIR's of $G$ up to projective equivalence, not up to similarity.

It is instructive at this point to apply the preceding analysis to two special cases which, in the next section, will turn out to correspond to the two cases where the geons are either all indistinguishable or all distinguishable, respectively.

Case I. Let the orbit $\mathcal{O}$ be such that the associated little group $K_{0}$ is all of $K$. Evidently, this is equivalent to saying that $\mathcal{O}$ is reduced to the single equivalence class $\left[\Gamma_{0}\right] \in \widehat{N}$. Then $R$ takes the form

$$
\begin{aligned}
& R(\dot{n})=\Gamma_{0}(\dot{n}) \otimes \mathbf{1}_{\beta}, \\
& R(\dot{k})=S(\dot{k}) \otimes T(\dot{k})
\end{aligned}
$$

(where we employ the notation $\dot{n}, \dot{k}$ to indicate that the equality holds for arbitrary elements $\dot{k} \in K, \dot{n} \in N)$. Recall that $T$ is a PUIR of $K$ and $S(k)$ is for each $k \in K$ an intertwining operator between $\Gamma_{0}(\dot{n})$ and $\Gamma_{0}\left(k \dot{n} k^{-1}\right)$. This UIR of $G$ is obviously finite dimensional if $S$ and $T$ are.

Case II. This case is the other extreme, where the orbit $\mathcal{O}$ is such that the little group associated with any element $\left[\Gamma_{0}\right] \in \mathcal{O}$ is trivial, i.e., $K_{0}=\{e\}$. In this case, $K / K_{0}=K /\{e\}$ 
is $K$ itself, $\mathcal{O}$ contains the maximum possible number $|K|$ of elements of $\widehat{N}(|K|$ being the order of $K$ ), and we have

$$
\widetilde{R}=\bigoplus_{i=0}^{|K|-1} \Gamma_{i}
$$

the multiplicity $\beta$ being one since the little group is trivial and has only the trivial representation. Clearly, $|K|$ must be finite in this case, since we have limited ourselves to finite dimensional representations $R$. Finally, we observe that since $T$ is trivial in the present case, $R(k)$ for $k \in K$ is composed entirely of intertwining maps connecting the various $\Gamma_{i}$. Moreover these maps must fit together without any phase mismatches, since the only possible PUIR of the little group $K_{0}=\{e\}$ is the trivial one, for which the multiplier $\sigma \equiv 1$.

Equipped in this manner, we now proceed to the physical situation at hand.

\section{$\S 4$. The UIR's that Annihilate the Slides}

A UIR $R: G \rightarrow A u t(\mathcal{H})$ which represents the slides trivially (annihilates them) is equivalent to a UIR of the quotient group $G / \mathcal{S} \simeq \widetilde{G}$, where $\widetilde{G}$ is the particle group defined earlier. But $\widetilde{G}$ is itself the semi-direct product $\widetilde{G}=G_{i n t} \ltimes S_{N}$, whence it lends itself naturally to the analysis of the preceding section. This will allow us to find explicitly all the UIR which annihilate the slides.

Now, in specializing the construction of $\S 3$ to the present situation we have $G \rightarrow \widetilde{G}$, $N \rightarrow G_{i n t}$ and $K \rightarrow S_{N}$. Here a crucial simplification occurs because $G_{i n t}$ is itself a product of smaller groups, indeed the direct product of $N$ copies of a single group $G_{1}$ : the MCG of the prime $\mathcal{P}$, or more precisely of $\mathcal{P} \# \mathbb{R}^{3}$. (Recall we have confined ourselves to 3 -manifolds of the form $\left.\mathbb{R}^{3} \# \mathcal{P} \# \mathcal{P} \# \cdots \# \mathcal{P}\right)$. Since $G_{\text {int }}$ is a direct product, its UIR's are the tensor product representations $\Delta_{1} \otimes \Delta_{2} \ldots \otimes \Delta_{N}$, where each $\Delta_{i}$ is some UIR of the internal diffeo group of a single prime. From this it follows that the operators $T(k)$ of $\S 3$ can be chosen canonically, and they will then manifestly furnish an ordinary representation of $K_{0}$ (i.e. one with $\sigma \equiv 1$ ). This follows from the fact that $T(k)$ just acts by permuting the factors of a tensor product, and such a permutation can always be implemented canonically - by an operator we will call $\mathcal{E}$. For example in $V \otimes V$ the exchange $\mathcal{E}$ taking $\psi_{1} \otimes \psi_{2} \rightarrow \psi_{2} \otimes \psi_{1}$ is the canonical choice, and cannot be mistaken for $\psi_{1} \otimes \psi_{2} \rightarrow-\psi_{2} \otimes \psi_{1}$. 
This uniqueness of the $\mathcal{E}$ 's is important for another reason. Not only does it

save us from having to consider projective representations in constructing $\widehat{\widetilde{G}}$ (because $\sigma \equiv 1 \Rightarrow \tau=1 / \sigma \equiv 1$ ), but with respect to physical interpretation, this absence of phaseambiguity will allow us to draw a meaningful distinction between the UIR's describing bosonic geons and those describing fermionic geons.

At this point we observe how the quantum behavior of otherwise identical primes can in fact render them distinguishable. We started off with $N$ classically identical primes whose internal groups $G_{i}$ are therefore all isomorphic. However these internal groups might all be represented by inequivalent UIR's $\Delta_{i}$. For example, if the internal group was generated by the $2 \pi$ rotation, i.e., $G_{i}=\left\{e, \mathcal{R}_{2 \pi}\right\} \simeq \mathbb{Z}_{2}$, then $\mathcal{R}_{2 \pi}$ could be represented either spinorially or tensorially [10]. Thus a generic UIR of the diffeo group of a set of such classically identical primes has both spinorial and tensorial geons, which accordingly belong to distinct species of particles. This then, is an example of what we referred to earlier as the quantum breaking of indistinguishability. (At the same time, one is led to the puzzle of what role the exchange operator plays when the geons are rendered distinguishable. We will comment on this later in the section).

To illustrate things more clearly, we consider the two simplest non-trivial cases, in which the manifold is made up of two and three identical primes respectively. One can readily infer from these examples what the structure of the UIR of $\widetilde{G}$ is for an arbitrary number of identical primes.

\section{Two identical Primes $(N=2)$}

Consider the situation where the three manifold $\mathcal{M}$ is $R^{3} \# \mathcal{P} \# \mathcal{P}$, where $\mathcal{P}$ is an irreducible prime. Then, $G_{\text {int }}=G_{1} \times G_{1}$ where the isomorphic internal groups $G_{1}$ and $G_{2}$ of the two primes have both been identified to $G_{1}$ (the particular choice of identification following from our choice of presentation of $\mathcal{M}$ ). The permutation subgroup is then $S_{2} \simeq$ $\mathbb{Z}_{2}$ and correspondingly, $\widetilde{G}=\left(G_{1} \times G_{1}\right) \ltimes \mathbb{Z}_{2}$, with $\mathbb{Z}_{2}$ acting on $G_{1} \times G_{1}$ by exchange of the $G_{1}$ factors. Let $R$ be a UIR of $\widetilde{G}$ and $\widetilde{R}$ its restriction to $G_{i n t}$. We must first find the orbits of the action of $S_{2}$ on the space $\widehat{G_{i n t}}$ of finite dimensional UIR's of $G_{i n t}$. These orbits can be classified into two types, namely (a) orbits corresponding to identical geons and (b) orbits corresponding to distinguishable geons. 
$\underline{\text { Type (a) orbit }}$

Here the fiducial element of our orbit is a UIR, $\Gamma \in \widehat{G}_{i n t}$ which has the form $\Gamma=\Delta \otimes \Delta$, where $\Delta$ is a UIR of $G_{1}$. (We omit the subscript 0 from $\Gamma$ since it is not needed in this case). The two primes therefore represent quantum mechanically identical geons. How does $S_{2}$ act on such a UIR? The only non trivial element of $S_{2}$ is the exchange $E$, and we observe that its action on $\Gamma$ is,

$$
\Gamma\left(g_{1}, g_{2}\right)=\Delta\left(g_{1}\right) \otimes \Delta\left(g_{2}\right) \mapsto \Gamma^{\prime}\left(g_{1}, g_{2}\right)=\Gamma\left(g_{2}, g_{1}\right)=\Delta\left(g_{2}\right) \otimes \Delta\left(g_{1}\right)
$$

But these two UIR's are equivalent to each other via the canonical exchange $\mathcal{E}(E)$ which just reverses the orders of the factors in the carrier space $\mathcal{H}_{\Delta} \otimes \mathcal{H}_{\Delta}$ of $\Delta \otimes \Delta$. Hence, the little group associated with the UIR $\Gamma$ is $S_{2}$ itself. This is an instance of case I of $\S 3$, so

$$
\widetilde{R}=(\Delta \otimes \Delta) \otimes \mathbf{1}_{\beta}
$$

with $\beta$ being the dimension of some UIR of $S_{2}$. Now section 3 tells us that $\widetilde{R}(k)=$ $S(k) \otimes T(k)$, where $k \in S_{2}$. But $S$ is just the (ordinary) representation of $S_{2}=\{1, E\}$ given by $S(1)=1$ and $S(E)=\mathcal{E}$, whence (since $S$ has trivial projective multiplier) $T$ must be an ordinary UIR of $S_{2}$. Hence $T$ is either trivial or is the 1-dimensional representation given by $T(E)=-1$ (with of course $T(1)=1$ ). Thus the full representation $R$ of $\widetilde{G}$ is given in this case by

$$
\begin{gathered}
\mathcal{H}=\mathcal{H}_{\Delta} \otimes \mathcal{H}_{\Delta} \\
\left(g_{1}, g_{2}\right) \mapsto \Delta\left(g_{1}\right) \otimes \Delta\left(g_{2}\right), \quad g_{i} \in G_{1}, \\
E \mapsto \pm \mathcal{E}
\end{gathered}
$$

where the two signs correspond to the two possibilities for $T$.

The physical interpretation for these two UIR's is evident. The geons are not merely identical classically, i.e. diffeomorphic, but are identical quantum mechanically; their internal groups are represented by the same UIR. Thus the exchange operator is physically meaningful, even at the quantum level, and this is reflected in the fact that $T$ can be a non-trivial representation of the permutation group, giving rise to the two different possible statistics, bosonic and fermionic. At this stage, one also sees the well known spin statistics violation emerging because of the possibility of arbitrary combinations of internal representations with statistics. In other words, given that the internal group is tensorially (spinorially) represented, i.e., $R_{2 \pi}$ is represented trivially (non-trivially), there is nothing 
to prevent the permutations from being fermionic (bosonic). Indeed we see that given any two identical primes, there exists at least one quantum sector for which the spin statistics correlation is violated.

Type (b) orbit

The other form that the fiducial UIR of the internal group $G_{i n t}$ can take is $\Gamma_{0}=$ $\Delta_{a} \otimes \Delta_{b}$ where $\Delta_{a}$ and $\Delta_{b}$ are inequivalent UIR's of the internal group of a single prime. Now the exchange $E$ has the action,

$$
\Delta_{a}\left(g_{1}\right) \otimes \Delta_{b}\left(g_{2}\right) \mapsto \Delta_{a}\left(g_{2}\right) \otimes \Delta_{b}\left(g_{1}\right) \simeq \Delta_{b}\left(g_{1}\right) \otimes \Delta_{a}\left(g_{2}\right)
$$

That is, $\Gamma_{0}=\Delta_{a} \otimes \Delta_{b}$ is taken into the inequivalent representation $\Gamma_{1}=\Delta_{b} \otimes \Delta_{a}$. Thus the little group associated with $\Gamma_{0}$ is trivial and one is in case II of $\S 3$ with $\mathcal{O}=$ $\left\{\Delta_{a} \otimes \Delta_{b}, \Delta_{b} \otimes \Delta_{a}\right\}$. Therefore (since the little group is trivial),

$$
\widetilde{R}=\Delta_{a} \otimes \Delta_{b} \oplus \Delta_{b} \otimes \Delta_{a}
$$

Using a block matrix notation, we can write

$$
\widetilde{R}=\left(\begin{array}{cc}
\Delta_{a} \otimes \Delta_{b} & 0 \\
0 & \Delta_{b} \otimes \Delta_{a}
\end{array}\right)
$$

and

$$
R(E)=\left(\begin{array}{cc}
0 & \mathcal{E}^{-1} \\
\mathcal{E} & 0
\end{array}\right)
$$

where now $\mathcal{E}: \mathcal{H}_{a} \otimes \mathcal{H}_{b} \rightarrow \mathcal{H}_{b} \otimes \mathcal{H}_{a}$ is the operator which takes $\psi_{a} \otimes \psi_{b} \mapsto \psi_{b} \otimes \psi_{a}\left(\mathcal{H}_{a}\right.$ being the carrier space of $\Delta_{a}$ and $\mathcal{H}_{b}$ that of $\Delta_{b}$ ). The form of this UIR of $\widetilde{G}$ is therefore completely determined.

In this case, we have a situation in which the geons are rendered distinguishable by the fact that their internal groups are represented inequivalently, and so the notion of statistics loses its meaning for this UIR because the operator $R(E)$ no longer has the significance of an exchange of identical objects. Consider for example a wave function in Hilbert space which is peaked at a point of $Q$ for which the primes have definite spatial locations with respect to a flat ambient geometry. For such states the representation $R$ gives direct information on the results of physical operations on the geons: exchange via parallel transport in the case of $E$, and local rotation or other "internal deformations" in the case of $G_{1}$ and $G_{2}$ (see [1], [2]). Let $\psi_{a b}$ be one such wave-function for which the geon 
at location 1 is "of species $a$ " (i.e., carries the representation $\Delta_{a}$ ) and that at location 2 is "of species b"; and let $\psi_{b a}=R(E) \psi_{a b}$ be the exchanged state. Clearly $\psi_{b a}$ is physically distinct from $\psi_{a b}$ (an example of "quantum multiplicity"), since for it the species of the geons at locations 1 and 2 have been swapped. Now, nothing stops us from forming the even and odd eigenvectors $\psi_{a b} \pm \psi_{b a}$ of $R(E)$, but the sign which occurs in these combinations has nothing to do with bose or fermi statistics, because the particles being "exchanged" are not identical. (See the discussion in $\S 6$ below, however.) It is exactly as if a proton and a neutron were present and $R(E)$ were the operator which exchanged their positions. That operator could not be used to determine nucleon statistics, and indeed it is entirely a matter of convention whether "the nucleon" is a boson or a fermion, as long as one has only a single $p-n$ system to refer to.

\section{Three Identical Primes $(N=3)$}

Let $\mathcal{M}=R^{3} \# \mathcal{P} \# \mathcal{P} \# \mathcal{P}$. This case is interesting because it is the simplest one in which non-abelian statistics (parastatistics) can occur. Moreover there are orbits in $\widehat{G_{\text {int }}}$ for which the little group is neither the whole permutation group $S_{3}$, nor trivial, and hence we need to use a greater portion of the apparatus developed in $\S 3$. The orbits in this case fall into three classes, (a) all three geons are identical, (b) all three geons are distinct, and (c) two geons are identical but distinct from the third. The analysis for orbit types (a) and (b) is similar to the what we saw in the $N=2$ case above, except that in case (a) the little group is $S_{3}$ rather than $S_{2}$, and therefore its UIR $T$ can be non-abelian as well as bosonic or fermionic (see equation (29) below). Thus not only is there a possibility of the violation of the usual spin-statistics correlation but also of the occurrence of parastatistics. Indeed, sectors manifesting bose, fermi and parastatistics exist for every irreducible prime $\mathcal{P}$ and there is nothing in the present analysis to rule out their appearance physically. We proceed to case $(\mathrm{c})$.

The fiducial UIR of the internal group for an orbit of type (c) has the form $\Gamma_{0}=$ $\Delta_{a} \otimes \Delta_{a} \otimes \Delta_{b}$. The little group associated with this UIR is the $\mathbb{Z}_{2}$ subgroup $K_{0}=\left\{e, P_{12}\right\}$, where by $P_{i j}$ we mean the exchange of the primes $i$ and $j$. Forming right cosets of $K_{0}$ in $S_{3}$ yields the partition,

$$
S_{3}=K_{0} \amalg K_{0} P_{13} \amalg K_{0} P_{23},
$$

so $\Gamma_{0}$ engenders an orbit of three elements given by the action of $P_{13}$ and $P_{23}$ on it. Therefore

$$
\widetilde{R}=\Delta_{a} \otimes \Delta_{a} \otimes \Delta_{b} \oplus \Delta_{b} \otimes \Delta_{a} \otimes \Delta_{a} \oplus \Delta_{a} \otimes \Delta_{b} \otimes \Delta_{a}
$$


since the UIR's of $\mathbb{Z}_{2}$ are one dimensional, whence the multiplicity $\beta$ is one. Since the little subgroup $K_{0} \subseteq S_{3}$ governs the statistics, and since $K_{0}=\mathbb{Z}_{2}$ has only a bosonic and a fermionic representation, we find again that the pair of identical geons can obey either bose or fermi statistics, again irrespective of whether their internal groups are represented spinorially or tensorially.

\section{The general case}

What can we infer from the above analysis for the general case of $N$ identical primes? If the fiducial UIR of the internal group $G_{i n t}$ is

$$
\Gamma_{0}=\underbrace{\Delta_{1} \otimes \cdots \otimes \Delta_{1}}_{N_{1}} \otimes \underbrace{\Delta_{2} \otimes \cdots \otimes \Delta_{2}}_{N_{2}} \cdots \otimes \underbrace{\Delta_{m} \otimes \cdots \otimes \Delta_{m}}_{N_{m}}
$$

where $N_{1}+N_{2}+\cdots+N_{m}=N$, then the little subgroup of $S_{N}$ associated with it will be $K_{0}=S_{N_{1}} \times S_{N_{2}} \times \cdots \times S_{N_{m}}$. To obtain a specific UIR $R$ of $\widetilde{G}$ we must then specify a UIR $T$ of $K_{0}$, or equivalently a UIR for each of its factor groups, $S_{N_{1}}, S_{N_{2}}, \ldots$, $S_{N_{m}}$. Thus the general UIR of $\widetilde{G}$ in the case of $N$ identical primes is given by the following:

- a choice of up to $N$ inequivalent UIR's $\Delta_{1}, \Delta_{2}, \ldots, \Delta_{m}$ of $G_{1}=\pi_{0}\left(\operatorname{Diff}^{\infty}\left(\mathcal{P} \# \mathbb{R}^{3}\right)\right)$

- For each $\Delta_{i}$ a multiplicity $\mathrm{N}_{i}$, such that $\Sigma N_{i}=N$.

- For each $\Delta_{i}$ a UIR $T_{i}$ of $S_{N_{i}}$, the permutation group on $N_{i}$ elements (i.e. a Young tableau with $N_{i}$ boxes).

Physically the resulting $R$ describes a quantum sector in which there are present $N_{i}$ geons of type $\Delta_{i}$ bearing the statistics defined by $T_{i}$. We see in particular that all possible parastatistics for a given $N$ can occur, and also that spin-statistics violating sectors will always exist for any set of identical irreducible primes.

The general case of an arbitrary (finite) collection of primes is an obvious generalization of the above, and need not be treated explicitly here. However we would like to stress that the quantum sectors which arise in the general case are neither more nor less than what would be suggested by the interpretation of geons as particles: a certain number of geons of each species is present and each species can have its own statistics. If there are any surprises or subtleties at this stage, they are associated only with the fact that the underlying prime manifold is not enough to determine a geon's species, but a UIR $\Delta$ of the prime's MCG is needed as well ("quantum breaking of indistinguishability"). In 
addition the complete decoupling of spin from statistics could seem surprising, but that is already an old story.

In addition to a classification of the UIR's it might be useful to have their full forms presented explicitly. Such a presentation in the general case seems too cumbersome to be illuminating, but the following two extreme cases are representative. They correspond to cases I and II of $\S 3$.

\section{All geons identical: case $\mathrm{I}^{\prime}$}

Here there is a single UIR $\Delta=\Delta_{1}$ of $G_{1}$, so $m=1, N_{1}=N$ and $K_{0}=S_{N}$, the full permutation group on $N$ elements. For $\left(g_{1}, g_{2} \ldots g_{N}\right) \in G_{i n t}=G_{1} \times G_{1} \times \ldots \times G_{1}$, we have from (16)

$$
R\left(\left(g_{1}, g_{2}, \ldots g_{N}\right)\right)=\left[\Delta\left(g_{1}\right) \otimes \ldots . \otimes \Delta\left(g_{N}\right)\right] \otimes \mathbf{1}_{\beta},
$$

where $\mathbf{1}_{\beta}$ is the identity operator in $\mathbb{C}^{\beta}$, while for $p \in S_{N}$, we have from (17)

$$
R(p)=\mathcal{E}(p) \otimes T(p)
$$

where $T$ is an arbitrary UIR of $S_{N}$ and $\mathcal{E}(p)$ is the canonical permutation of the factors of $\mathcal{H}_{\Delta} \otimes \mathcal{H}_{\Delta} \otimes \cdots \otimes \mathcal{H}_{\Delta}, \mathcal{H}_{\Delta}$ being the carrier space of $\Delta$.

\section{All geons distinct: case $\mathrm{II}^{\prime}$}

Here all the $\Delta_{i}$ are distinct, so $N_{1}=N_{2}=\cdots=N_{N}=1, K_{0}=\{e\}$ and

$$
\widetilde{R}=\left[\Delta_{1} \otimes \Delta_{2} \cdots \otimes \Delta_{N}\right] \oplus\left[\Delta_{2} \otimes \Delta_{1} \cdots \otimes \Delta_{N}\right] \oplus \cdots \cdots
$$

as in (18), while

$$
R(p)=\mathcal{E}(p)
$$

As in the analogous cases above for 2 and 3 primes, no notion of statistics enters here since the geons are all distinct.

\section{$\S 5$. The General UIR of $G$ and Two Special Cases}

In the preceding section we have seen that the structures which appeared in the general analysis of $\S 3$ have a direct physical meaning when $G$ is the particle group, $\widetilde{G}=$ $G_{\text {int }} \ltimes S_{N}$. As applied to $\widetilde{G}$, that analysis told us that the general (finite dimensional) UIR of $\widetilde{G}$ can be obtained from a UIR of $G_{i n t}$ together with a UIR of the corresponding little

group $K_{0} \subseteq S_{N}$. Physically the UIR of $G_{i n t}$ (or rather its $S_{N}$-orbit in $\widehat{G_{i n t}}$ ) determines the species of the geons which are present, while the UIR of $K_{0}$ determines their statistics. 
The above analysis covers all those UIR's of $G=\pi_{0}\left(\operatorname{Diff}^{\infty}(\mathcal{M})\right)$ for which the slide subgroup $\mathcal{S}$ is represented trivially. If we drop this restriction, then we obtain a more complicated situation in which different decompositions of $G$ are possible. Now, we have a nested triad of normal subgroups,

$$
\mathcal{S} \subseteq \mathcal{S} G_{i n t} \subseteq \mathcal{S} G_{i n t} S_{N}=\mathcal{S} \widetilde{G}=G
$$

and the analysis of $\S 3$ can be applied in stages. One possibility would be to build up $G$ as

$$
G=\left(\mathcal{S} \ltimes G_{i n t}\right) \ltimes S_{N},
$$

which would lead to first finding the UIR's of $\mathcal{S}$, then those of $\mathcal{S} \ltimes G_{\text {int }}$, and finally those of $G$. If in the process a proper subgroup of $G_{i n t}$ emerged as a little group, we could speak of a "quantum breaking of internal symmetry". Similarly, if a proper subgroup of $S_{N}$ appeared as a little group at the next stage, we could speak of a "quantum breaking of indistinguishability" as before. A related scheme would analyze $G$ as

$$
G=\mathcal{S} \ltimes \widetilde{G}=\mathcal{S} \ltimes\left(G_{\text {int }} \ltimes S_{N}\right)
$$

in which case we would obtain a single little group as a subgroup of $\widetilde{G}=G_{i n t} \ltimes S_{N}$ rather than a pair of little groups in $G_{i n t}$ and $S_{N}$ separately. The resulting description of a given $R \in \widehat{G}$ would not appear to differ significantly from that tied to the decomposition (33).

Once one had obtained a UIR $R$ of $G$, it could also prove informative to decompose it into a sum of UIR's of $\widetilde{G}=G_{i n t} S_{N}$, rather than of $\mathcal{S}$ or $\mathcal{S} G_{\text {int }}$. Although such a decomposition is not of the type studied in $\S 3$ (because $\widetilde{G}$ is not normal in $G$ ), it could often be the most appropriate physically. Decomposing $R$ in this way would lead to a description in which the slides did not break internal symmetries or geon distinguishability, but rather induced transitions between different types of geons (inelastic scattering). We suspect that the different languages associated with these different ways of decomposing $R$ might all be useful depending on the physical situation under study. For example the decomposition corresponding to $\mathcal{S} \subseteq \mathcal{S} G_{\text {int }} \subseteq G$ might be suitable for a hot dense "gas" of geons, whereas that corresponding to $\widetilde{G} \subseteq G$ might be best for talking about geon-geon scattering.

In any case, none of these contemplated analyzes can be completed without an understanding of the space $\widehat{\mathcal{S}}$ of UIR's of $\mathcal{S}$, or at least some understanding of what types of little groups are induced by the action of $\widetilde{G}$ on $\widehat{\mathcal{S}}$. In addressing this question, one has 
available (from §2) the knowledge of how the permutations and the internal diffeomorphisms act on a generating set of slides, and in principle this is all we would need to know in determining the $G_{\text {int }}$ or $\widetilde{G}$ orbits in $\widehat{\mathcal{S}}$.

Let us remark here that the subgroup of the diffeos consisting of only the $2 \pi$ rotations, which we shall call $\mathcal{R}_{2 \pi}$, in fact commutes with all diffeos and hence will always be in (the center of) the little group of any element of $\widehat{\mathcal{S}}$

Unfortunately, an analysis of $\widehat{G}$ as complete as that of $\widehat{\widetilde{G}}$ in $\S 4$ seems out of reach. To start with, the UIR's of $\mathcal{S}$ are much harder to characterize than were those of $G_{i n t}$, an important difference being that $G_{i n t}$ is a direct product whereas $\mathcal{S}$ in general involves free products. Similarly, the action of $\widetilde{G}$ on $\mathcal{S}$ is more complicated than was that of $S_{N}$ on $G_{i n t}$. Moreover, the seeming lack of a canonical choice for little group operators of the type $S\left(g_{\text {int }}\right)$ or $S(p)$ means that nontrivial projective multipliers can be expected to come into play when the slides are nontrivially represented. For all these reasons we are unable to offer complete results in the general case. Nevertheless, there are still special situations in which the analysis can be carried through, and already in these special cases we will encounter interestingly novel types of particle behavior. In the remainder of this section we consider two such situations.

$\underline{\text { Case }(\mathcal{A})}$ Let $\mathcal{P}$ be a prime whose internal group is trivial $\left(\mathcal{P} \simeq \mathbb{R} P^{3}\right)$. Then $G_{\text {int }}=\{e\}$ and we have simply $G=\mathcal{S} \ltimes S_{N}$. Further, let us select an orbit $\mathcal{O}$ of the $S_{N}$ action on $\widehat{\mathcal{S}}$ whose associated little group is also trivial. We are then in case II of $\S 3$ (with $\left.N \rightarrow \mathcal{S}, K \rightarrow S_{N}\right) ; \mathcal{O}$ is essentially a copy of $S_{N}$ itself, and we acquire from $\mathcal{O}$ a unique UIR $R$ of $G$, for which

$$
\widetilde{R} \equiv R \mid \mathcal{S}=\Gamma_{0} \oplus \Gamma_{1} \oplus \cdots \oplus \Gamma_{m-1}
$$

where $m=N$ ! is the order of $S_{N}$ and the $\Gamma_{i}$ are the elements of $\mathcal{O}$. Since the little subgroup of $S_{N}$ is trivial, the UIR $T$ is also trivial, and the possibility of different types of statistics does not arise.

In $\S 4$ we interpreted a similar situation to mean that the geons were quantum mechanically inequivalent because their internal groups were represented differently. However in the present case the internal groups are all trivial and hence represented identically. What can the inequivalence of the UIR's $\Gamma_{j}$ mean physically in this case? Consider an element of $S_{N}$, say the exchange $p_{i j}$ of the $i^{t h}$ with the $j^{\text {th }}$ prime. Since the little group associated with the representation $\Gamma_{0}$ is trivial, $\Gamma_{0} \mapsto \Gamma_{k} \nsucceq \Gamma_{0}$ under the action of $p_{i j}$. Now, $p_{i j}$ will commute with all slides that do not involve the $i^{\text {th }}$ or the $j^{\text {th }}$ prime. Thus 
if $\Gamma_{k}$ is inequivalent to $\Gamma_{0}$, it can only mean, for example, that the slide of the $i^{\text {th }}$ geon through the $j^{\text {th }}$ is not the same, physically, as that of the $j^{\text {th }}$ geon through the $i^{\text {th }}$. We encounter here a quantum effect unlike that in any other system to our knowledge, namely that slides (essentially a type of collision) render distinguishable particles which cannot be told apart if examined separately. In other words, if one merely examined the individual properties of these geons, they would seem to be identical, but if they were made to interact appropriately with each other, one would find that they were in fact different. We will encounter a concrete example of this effect in $\S 6$.

$\underline{\text { Case }(\mathcal{B})}$ The situation just discussed manifested a "quantum breaking" of permutation symmetry. A different possibility is that the permutations remain intact while the internal symmetry is broken. To see how this might occur, consider the decomposition $G=\mathcal{S} \ltimes \widetilde{G}$ (with $K:=\widetilde{G}=G_{i n t} \ltimes S_{N}$ ) and suppose that $\Gamma_{0}$ is a UIR of $\mathcal{S}$ for which the little subgroup $K_{0} \subseteq \widetilde{G}$ is reduced to $K_{0}=S_{N}$. (This implies that $\mathcal{R}_{2 \pi}$ must be trivial as remarked earlier). Since $\widetilde{G}=G_{i n t} \ltimes S_{N}$, the coset space $K_{0} \backslash K=S_{N} \backslash \widetilde{G}$ can be identified with $G_{\text {int }}$ and we have

$$
\widetilde{G}=\amalg_{\alpha} S_{N} b_{\alpha},
$$

where the $b_{\alpha}$ run through $G_{i n t}$ as $\alpha$ runs from 0 to $m-1$, with $m=\left|G_{i n t}\right|$. (Note however that $S_{N} \backslash \widetilde{G}$ is not a group since $S_{N}$ is not normal in $\widetilde{G}$ ). The "core" representation thus takes the form

$$
S \otimes T
$$

where $S$ is a PUIR acting on the carrier space of $\Gamma_{0}$ and $T$ is a PUIR of $S_{N}$ of dimension $\beta$ which we can interpret as determining the statistics of the geons. [We know of no reason why $S$, and therefore $T$, would not be properly projective in general. This would produce a new kind of "generalized statistics" determined by a projective representation of the permutation group rather than an ordinary one. (Non-trivial projective representations of $S_{N}$ exist for $N \geq 4$.)]. It follows that

$$
\widetilde{R}=\left[\Gamma_{0} \oplus \cdots \Gamma_{m-1}\right] \otimes \mathbf{1}_{\beta}
$$

and the dimension of our UIR is $m \beta\left(\operatorname{dim} \Gamma_{0}\right.$ ). (So we need $\left|G_{i n t}\right|<\infty$, in order to comply with our standing restriction to finite dimensional representations).

Since the little group is all of $S_{N}$ in this type of example, the geons must all be identical. What does it mean, however, that $G_{i n t}$ is not part of the little group? Classically 
an internal diffeo corresponds to an internal symmetry of a prime $\mathcal{P}$. If such a diffeo takes a generator $\phi \in \pi_{1}(\mathcal{P})$ to a generator $\phi^{\prime}$, then this means that $\phi$ and $\phi^{\prime}$ are in a sense equivalent. On quantization, however, if the UIR's $\Gamma_{i}$ are not left invariant under the action of $G_{\text {int }}$ (as in (38)), then this must mean that the slides of some other prime along the two generators are inequivalent. In other words, the slides have rendered the geons "asymmetric"! Again we observe that merely examining an isolated geon is not enough to determine whether it is symmetric. One requires "collisions" with the other geons in order to determine whether the classical internal symmetry has been broken or not. This type of symmetry breaking also appears to be novel.

In the next section we will construct examples of case $\mathcal{A}$ where $\mathcal{M}$ is the connected sum of $\mathbb{R}^{3}$ with $N$ copies of $\mathbb{R} P^{3}$, and where $\Gamma_{0}$ is an abelian UIR of $\mathcal{S}$. One can also construct examples of case $\mathcal{B}$ in a similar manner, but since we lack a systematic analysis, we will not present any explicit examples of case $\mathcal{B}$.

\section{$\S 6$. The Example of $\mathbb{R} P^{3}$ Geons}

Let the manifold $\mathcal{M}=\mathbb{R}^{3} \# \underbrace{\mathbb{R} P^{3} \cdots \# \mathbb{R} P^{3}}_{N}$. (The connected sum of $\mathbb{R}^{3}$ with $N$ $\mathbb{R} P^{3}$ primes can be visualized as follows: take $\mathbb{R}^{3}$ and remove $N$ three balls $\mathbf{D}^{3}$ from it; then perform an antipodal identification for each of the resulting $S^{2}$ boundaries.) The internal diffeo group for an $\mathbb{R} P^{3}$ prime is trivial, and therefore the MCG of $\mathcal{M}$ is $\mathcal{S} \ltimes S_{N}$. Moreover, $\pi_{1}\left(\mathbb{R} P^{3}\right)=\mathbb{Z}_{2} \Rightarrow s^{2}=e$ for any slide $s$. The slide subgroup therefore has $N(N-1)$ generators $s_{i j}$ satisfying $s_{i j}^{2}=e$ and satisfying the Fuks-Rabinovitch commutation relations given in $\S 2$. Notice that the latter become entirely trivial for any 1-dimensional representation of the slides.

\section{$\underline{\text { A pair of } \mathbb{R} P^{3} \text { geons }}$}

The mapping class group $G=\pi_{0}\left(\operatorname{Diff}\left(\mathbb{R}^{3} \# \mathbb{R} P^{3} \# \mathbb{R} P^{3}\right)\right)$ is the semidirect product $G=\mathcal{S} \ltimes S_{2}$, where the nontrivial element $E \in S_{2}$ interchanges the two primes, and the slide subgroup, $\mathcal{S}=\mathbb{Z}_{2} * \mathbb{Z}_{2}$, is generated by the slide $s_{1}$ of the first prime through the second together with the slide $s_{2}$ of the second prime through the first [6] [21]. Clearly $E$ acts on the normal subgroup $\mathcal{S}$ by exchanging its two $\mathbb{Z}_{2}$ factors. A set of generators and relations for $G$ can then be given as follows.

$$
G=<s_{1}, s_{2}, E: s_{1}^{2}=s_{2}^{2}=E^{2}=1, E s_{1}=s_{2} E>
$$


Notice that not only $\mathcal{S}$ but $G$ itself is isomorphic to $\mathbb{Z}_{2} * \mathbb{Z}_{2}$, as can be seen from the following alternative presentation.

$$
G=<x, y: x^{2}=y^{2}=1>
$$

where $x=s_{1}$ and $y=E$. (Of course an infinite group can be isomorphic to a proper subgroup without contradiction.)

The presentation (40) reduces the problem of finding the UIR's of $G$ to that of finding the UIR's of $\mathbb{Z}_{2} * \mathbb{Z}_{2}$. This could be done directly (and has been in [2]), however for present purposes it is more instructive to build up the UIR's of $G$ from those of $\mathcal{S}$ and $S_{2}$ by applying the method of $\S 3$ with $N \rightarrow \mathcal{S}, K \rightarrow S_{2}$. In this way, we will be able to identify the appropriate little groups in each case, enabling us to draw conclusions about geon indistinguishability and statistics.

Following the pattern of $\S 3$, our first step is to determine the UIR's of $\mathcal{S}$ and the orbits $\mathcal{O}$ induced in $\mathcal{S}$ by the action of $S_{2}$. The UIR's of $\mathcal{S} \simeq \mathbb{Z}_{2} * \mathbb{Z}_{2}$ may themselves be found by the method of $\S 3$, taking for the normal subgroup $N^{\prime} \subseteq \mathbb{Z}_{2} * \mathbb{Z}_{2}$ the cyclic subgroup $\left\{(x y)^{n} \mid n \in \mathbb{Z}\right\} \simeq \mathbb{Z}$. With this identification, $N^{\prime}$ is abelian and its character group $\widehat{N^{\prime}}$ contains elements $\Gamma_{\theta}$ parameterized by an angle $\theta \in \mathbb{R} \bmod 2 \pi$ (i.e. $\Gamma_{\theta}(n)=q^{n}$ with $\left.q=e^{2 \pi i \theta}\right)$. Under the action of $\mathbb{Z}_{2}=\mathbb{Z}_{2} * \mathbb{Z}_{2} / N^{\prime}$, there are a pair of singleton orbits $\mathcal{O}=\left\{\Gamma_{0}\right\}, \mathcal{O}=\left\{\Gamma_{\pi}\right\}$ and an open interval's worth of 2-element orbits $\mathcal{O}=\left\{\Gamma_{\theta}, \Gamma_{-\theta}\right\}$, where $0<\theta, \pi$. The corresponding UIR's are readily found, and are listed, in a symmetrically chosen basis, in Table 1 (wherein the parameters $\tau$ and $\gamma$ are respectively $\cos \theta / 2$ and $\sin \theta / 2$, and the first four UIR's correspond to $\theta=0$ and $\theta=\pi)$. Having now determined the space $\widehat{\mathcal{S}}$, our next step is to fiber it into orbits $\mathcal{O}$ under the action of $S_{2}=G / \mathcal{S}$.

Under exchange of the slides $s_{1}$ and $s_{2}$, the UIR's in table 1 fall into orbits of four types. The first two UIR's are evidently fixed points, and hence each constitutes an orbit in itself. Similarly, the third and fourth UIR's combine to form a single orbit. Finally, each 2-dimensional UIR is also a fixed point, since the exchange can be implemented unitarily by the operator $S(E):=\left(\begin{array}{cc}1 & 0 \\ 0 & -1\end{array}\right)$. Taking these cases in order, we get the following decomposition of $\widehat{\mathcal{S}}$ into disjoint orbits (using the names given in table 1 ):

$$
\widehat{\mathcal{S}}=\left\{\Gamma_{++}\right\} \cup\left\{\Gamma_{--}\right\} \cup\left\{\Gamma_{+-}, \Gamma_{-+}\right\} \cup \bigcup_{0<\tau<1}\left\{\Gamma_{\tau}\right\}
$$


Evidently the associated little groups $K_{0} \subseteq S_{2}=\mathbb{Z}_{2}$ are

$$
K_{0}=\mathbb{Z}_{2}, \quad K_{0}=\mathbb{Z}_{2}, \quad K_{0}=\{e\}, \quad K_{0}=\mathbb{Z}_{2}
$$

and for the representations $S$ of $K_{0}$, we may choose

$$
S(E)=1, \quad S(E)=1, \quad S=1, \quad S(E)=\left(\begin{array}{cc}
1 & 0 \\
0 & -1
\end{array}\right)
$$

(Here the first three choices of $S(E)$ are trivial of course, since all the UIR's involved are 1-dimensional). Given these orbits, little groups and operators $S(E)$, we can proceed to construct the UIR's of $G=\mathcal{S} \ltimes S_{2}$.

Table 1: UIR's of the slide subgroup $\mathbb{Z}_{2} * \mathbb{Z}_{2}$ The final column shows the "Casimir invariant" $\frac{1}{2} \Gamma\left(s_{1} s_{2}+s_{2} s_{1}\right)$

\begin{tabular}{cccll}
\hline \hline $\operatorname{Dim}$ & $\Gamma\left(s_{1}\right)$ & $\Gamma\left(s_{2}\right)$ & Name & Invariant \\
\hline 1 & 1 & 1 & $\Gamma_{++}$ & +1 \\
1 & -1 & -1 & $\Gamma_{--}$ & +1 \\
1 & 1 & -1 & $\Gamma_{+-}$ & -1 \\
1 & -1 & 1 & $\Gamma_{-+}$ & -1 \\
2 & $\left(\begin{array}{cc}\tau & \gamma \\
\gamma & -\tau\end{array}\right)$ & $\left(\begin{array}{cc}\tau & -\gamma \\
-\gamma & -\tau\end{array}\right)$ & $\Gamma_{\tau}$ & $2 \tau^{2}-1$ \\
& $\tau \in(0,1)$ & $\gamma=\sqrt{1-\tau^{2}}>0$ & & \\
\hline \hline
\end{tabular}

The orbits $\left\{\Gamma_{++}\right\}$and $\left\{\Gamma_{--}\right\}$

Both of these cases are essentially trivial since $\Gamma$ is 1-dimensional and the little group $K_{0}$ of (42) is $\mathbb{Z}_{2}$, which has only the two 1-dimensional UIR's $T(e)=1, T(E)= \pm 1$. (Projective multipliers obviously do not arise in this situation, since $\sigma \equiv 1$ for the trivial representations $S$ of (43).) Since $\mathcal{O}$ consists of a single point, our "core" representation (11) is already all of $R$, and we obtain the $2 \times 2=4$ abelian UIR's of $G$, which are listed in Table 2. (The first and third come from $\Gamma_{++}$, the second and fourth from $\Gamma_{--}$.) 
Since the little group in these two cases is $S_{2}$, we may interpret these UIR's as describing identical geons with statistics given by $T(E)$. This obviously makes physical sense since $R(E)$ is a simple sign in each case. The first two representations in Table 2 describe bosonic geons, and the second two describe fermionic ones.

The orbit $\left\{\Gamma_{+-}, \Gamma_{-+}\right\}$

Here the little group is trivial and so (see eq. (18) above)

$$
\widetilde{R}=\Gamma_{+-} \oplus \Gamma_{-+},
$$

or in a matrix notation,

$$
\begin{aligned}
& R\left(s_{1}\right)=\left(\begin{array}{cc}
1 & 0 \\
0 & -1
\end{array}\right), \\
& R\left(s_{2}\right)=\left(\begin{array}{cc}
-1 & 0 \\
0 & 1
\end{array}\right) .
\end{aligned}
$$

The exchange operator then transforms (44) into (45):

$$
R(E)=\left(\begin{array}{ll}
0 & 1 \\
1 & 0
\end{array}\right) .
$$

\begin{tabular}{|c|c|c|c|c|}
\hline Dim & & $R\left(s_{1}\right)$ & $R\left(s_{2}\right)$ & $R(E)$ \\
\hline 1 & & 1 & 1 & 1 \\
\hline 1 & & -1 & -1 & 1 \\
\hline 1 & & 1 & 1 & -1 \\
\hline 1 & & -1 & -1 & -1 \\
\hline 2 & & $\left(\begin{array}{cc}1 & 0 \\
0 & -1\end{array}\right)$ & $\left(\begin{array}{cc}-1 & 0 \\
0 & 1\end{array}\right)$ & $\left(\begin{array}{ll}0 & 1 \\
1 & 0\end{array}\right)$ \\
\hline 2 & $\begin{array}{l}\tau \in(0,1) \\
\gamma=\sqrt{1-\tau^{2}}>0\end{array}$ & $\left(\begin{array}{cc}\tau & \gamma \\
\gamma & -\tau\end{array}\right)$ & $\left(\begin{array}{cc}\tau & -\gamma \\
-\gamma & -\tau\end{array}\right)$ & $\pm\left(\begin{array}{cc}1 & 0 \\
0 & -1\end{array}\right)$ \\
\hline
\end{tabular}

This non-abelian UIR of $G$ furnishes the fifth row of table 2 .

Table 2: UIR's of the mapping class group $G$ for two $\mathbb{R} P^{3}$ geons 
Since the little subgroup of $S_{2}$ is trivial in this case, we may interpret the corresponding UIR as describing non-identical geons. Hence, the question of statistics does not arise with this type of interpretation, as discussed earlier in $\S \S 4$ and 5. Observe, however, that in this case the geons are indistinguishable in themselves. It is only the manner in which they slide through each other that differentiates them (specifically the relative minus sign in lines 3 and 4 of Table 1 ).

For future reference, we note here that it is possible to diagonalize $R(E)$ by a simple rotation. Doing so brings our representation to the form

$$
R\left(s_{1}\right)=\left(\begin{array}{ll}
0 & 1 \\
1 & 0
\end{array}\right)=-R\left(s_{2}\right), \quad R(E)=\left(\begin{array}{cc}
1 & 0 \\
0 & -1
\end{array}\right)
$$

The orbits $\left\{\Gamma_{\tau}\right\}, 0<\tau<1$

Once again, the little group $K_{0}$ of (42) is $S_{2}=\mathbb{Z}_{2}$ and $\mathcal{O}$ consists of a single element $\Gamma=\Gamma_{\tau}$. Hence the "core" representation (111) is again the whole of $R$. Now however, $\Gamma$ is 2 -dimensional and more of the machinery of $\S 3$ comes into play. With our choice of

$$
S(E)=\left(\begin{array}{cc}
1 & 0 \\
0 & -1
\end{array}\right)
$$

the projective multiplier $\sigma$ is trivial (as it must be for $\mathbb{Z}_{2}$ ) and $R$ is determined by a UIR $T$ of $K_{0}=\mathbb{Z}_{2}$, or in other words by a sign:

$$
T(E)= \pm 1 .
$$

The resulting representation $R$ is then given (since $T$ is a 1-dimensional representation) by

$$
\begin{gathered}
R\left(s_{i}\right)=\Gamma\left(s_{i}\right) \otimes \mathbf{1}_{1}=\Gamma\left(s_{i}\right) \quad(i=1,2) \\
R(E)=S(E) \otimes T(E)=S(E) T(E)= \pm S(E)
\end{gathered}
$$

or in our chosen basis

$$
R(E)= \pm\left(\begin{array}{cc}
1 & 0 \\
0 & -1
\end{array}\right)
$$

with $R\left(s_{i}\right)$ as in Table 1 . This pair of UIR's provides the last line of Table 2, thereby completing our construction of $\widehat{G}$.

What shall we say about statistics in this case? From the little group's being $S_{2}$ we may conclude that the geons are identical. Moreover following $\S 4$, we would naturally 
associate the statistics of the geons with the representation $T$ of $S_{2}$. That is, we would be tempted to say that the plus sign in (49) describes bosons and the minus sign fermions. However, such an identification appears to suffer from an ambiguity not present in the case of the particle group $\widetilde{G}$. There we could make a canonical choice of the UIR $S$, based on the fact that $\Gamma_{0}$ was a tensor product of 1-geon UIR's. Here $\Gamma_{\tau}$ lacks such extra structure, and there seems no good reason why we couldn't equally well reverse the sign in our choice of $S(E)$ in (48), thereby interchanging the designations "fermionic" and "bosonic". (It is true that we can "tell the difference" between the two choices, but what seems lacking is any grounds for singling out either choice of $S(E)$ as yielding "simple exchange without a minus sign".) In the face of this ambiguity, it may be best just to say that the two signs in the last line of Table 2 express an alternative analogous to the bose-fermi one, but not necessarily identifiable with it.

The confusion is heightened if we ask ourselves how the UIR's of Table 2 correspond to those of Table 1 (after all, both the groups $G$ and $\mathcal{S}$ are isomorphic to $\mathbb{Z}_{2} * \mathbb{Z}_{2}$ ). For the 1-dimensional representations the correspondence is easy (just omit the $s_{2}$-column from Table 2 to obtain Table 1), but for the 2-dimensional representations the correspondence is less obvious because there are three families of representations in Table 2 and only one in Table 1. To reveal the correspondence, it suffices to rewrite the representations in Table 2. First notice that the sign in $R(E)$ can be swapped for a sign in $\tau$, which then ranges over $(-1,1) \backslash\{0\}$. The penultimate line in Table 2 then provides the missing $\tau=0$ case of this family, if we write it in the form (47). Thus the entire set of two-dimensional representations of Table 2 can be seen to make up an open interval's worth of UIR's, exactly as in Table 1. The only difference is that in Table 2 the interval is parameterized by $\tau \in(-1,1)$, instead of by $\tau \in(0,1)$. From this point of view, the two-dimensional representations of $G$ are drawn from a single continuum, and it seems peculiar that one point of that continuum should describe distinguishable geons, when all the others describe identical ones.

$\underline{\text { An alternative viewpoint }}$

In fact there exists an alternative point of view (hinted at in $\S 5$ ), from which the geons are identical in all cases, and it may be that this view is the more appropriate one in some situations. Indeed, let us return for a moment to the $p-n$ system which, we argued earlier, was analogous to a pair of identical primes carrying inequivalent representations of the 1-geon MCG. Formally, it would be possible to regard the exchange of $p$ with $n$ 
as an exchange of identical particles, but then both types of statistics would be present simultaneously, depending on the parity of the center of mass wave function. ${ }^{*}$ Processes which changed this parity would be regarded as changing the statistics of the particles in effect changing their identity. Such a point of view would seem to be rather useless for an actual $p-n$ system, but it might make more sense if it were very difficult in practice to distinguish a proton from a neutron (thus, if processes mixing our putative "bosonic" and "fermionic" states occurred on longer time scales than processes taking place within these subspaces). Indeed, a description very much like this one is common in talking about ortho- and para-hydrogen as if they were composed of different kinds of constituents.

Now in the case of slides, one can imagine situations (a dilute gas of geons, for instance, or geon-geon scattering at low energies) where exchanges of location occur much more frequently than collisions in which one geon penetrates the other. In such a situation, each 2-dimensional representation of table 2 would effectively decouple into a pair of 1-dimensional subrepresentations, corresponding to $R(E)= \pm 1$. The $R(E)=+1$ subspace could then be interpreted naturally as describing identical bosonic geons, the other subspace identical fermionic geons, and transitions between the two subspaces would be induced (relatively infrequently) only by special kinds of inelastic collisions (slides). Within this interpretation, moreover, all the 2-dimensional representations of Table 2 would be on the same footing, nor would we be using "nonlocal" effects (the slides) to determine geon identity. Depending on the dynamical situation, such a viewpoint might sometimes be preferable to the more "kinematical" one we have adopted in the present paper. On the other hand, the method we have adopted for defining particle identity and statistics is strongly suggested by the mathematics of $\S 3$; and, as we have seen, it convincingly interprets the representations in which the slides act trivially $(\S 4)$. Here, we wished only to raise the possibility of an alternative description. In the sequel we will stick, for clarity, to our standard mode of expression, in which particle identity and statistics are to be deduced from the little subgroup $K_{0} \subseteq S_{N}$ and its PUIR $T$. The difference in any case is only one

* Here we are not referring to the common practice of regarding proton and neutron as different isospin states of a single particle, the nucleon. That would be to embed the $p-n$ statespace $\mathcal{H}$ in a larger state-space including the $p-p$ and $n-n$ systems as well as the $p-n$. Rather we mean to leave $\mathcal{H}$ unchanged but pretend that the proton and neutron are indistinguishable, defining bose and fermi sectors of $\mathcal{H}$ with respect to the operator which exchanges the (space and spin) coordinates of $p$ and $n$. 
of convenience; it is the representation $R$ as a whole that determines the physics, not its decomposition into one or another type of sub-representation.

\section{Three $\mathbb{R} P^{3}$ geons}

The mapping class group when $N=3$ is $G=\mathcal{S} \ltimes S_{3}$. Now, $\mathcal{S}$ is generated by $3 \times 2=6$ slides $s_{i}^{j}$ satisfying the commutation relations quoted in $\S 2$, which here reduce to $s_{i}^{j} \natural s_{k}^{j}$ and $s_{i}^{j} s_{k}^{j} \natural s_{i}^{k}$. Although we cannot offer a full classification in this case, one can certainly find some UIR's of $\mathcal{S}$ and use them to construct UIR's of $G$.

We list in table 3 the UIR's of $G$ which result from the simplest abelian UIR's $\Gamma$ of $\mathcal{S}$. (Notice that for abelian $\Gamma$, the PUIR $S$ of $K_{0}$ is trivial; hence there is no phase ambiguity in the UIR $T$ of $K_{0}$, and our identification of the geon statistics resulting from a given choice of $T$ is correspondingly unambiguous.) For example, in (a) we have taken the trivial representation of $\mathcal{S}$, and hence the corresponding little group is $K_{0}=S_{3}$; then all the geons are identical and can either obey fermi, bose or parastatistics. For all the other UIR's listed in the table, with the exception of $(\mathrm{j})$, the slides render the geons wholly or partly distinct; either all three are different $\left(K_{0}=\{e\}\right)$ or two are the same but differ from the third $\left(K_{0}=S_{2}\right)$. In (j) however, $K_{0}=\mathbb{Z}_{3}$ implying that in this case statistics is determined not by an $S_{n}$ subgroup of $S_{3}$, but by $\mathbb{Z}_{3} \subseteq S_{3}$, the subgroup of cyclic permutations. In words: the geons are invariant only under cyclic permutations but not under a simple exchange, they are "cyclically identical" but not pairwise identical! One is reminded of other situations in physics in which statistics is determined by a nonpermutation group, for example the braid group in the case of identical particles on a plane. 
Table 3: Some UIR's for three $\mathbb{R} P^{3}$ geons, from abelian UIR's of the slides

In the first column, all unspecified $\Gamma_{0}\left(s_{i}{ }^{j}\right)=1$

\begin{tabular}{|c|c|c|c|c|c|c|}
\hline & $\begin{array}{l}\text { Fiducial } \\
\text { UIR of } \mathcal{S} \\
\Gamma_{0}\end{array}$ & $\begin{array}{l}\text { Little } \\
\text { group } \\
K_{0}\end{array}$ & $\begin{array}{l}\nu= \\
\left|S_{3} / K_{0}\right| \\
=|\mathcal{O}|\end{array}$ & $\begin{array}{l}\text { UIR } T \\
\text { of } K_{0}\end{array}$ & $\begin{array}{l}\widetilde{R}= \\
\left(\bigoplus_{i=0}^{\nu-1} \Gamma_{i}\right) \otimes \mathbf{1}_{\beta}\end{array}$ & $\begin{array}{l}\text { Identity: } \\
\text { Statistics }\end{array}$ \\
\hline (a) & trivial & $S_{3}$ & 1 & $\begin{array}{ll}2 & 1-d \\
1 & 2-d\end{array}$ & $\mathbf{1}_{\beta}$ & $\begin{array}{l}\text { all identical: } \\
\text { Fermi, Bose, } \\
\text { para }\end{array}$ \\
\hline (b) & $\Gamma_{0}\left(s_{3}^{2}\right)=-1$ & $\{e\}$ & 6 & trivial & $\bigoplus_{i=0}^{5} \Gamma_{i}$ & all distinct \\
\hline (c) & $\begin{array}{l}\Gamma_{0}\left(s_{3}{ }^{1}\right) \\
\Gamma_{0}\left(s_{1}^{2}\right)=-1\end{array}$ & $\{e\}$ & 6 & trivial & $\bigoplus_{i=0}^{5} \Gamma_{i}$ & all distinct \\
\hline (d) & $\begin{array}{l}\Gamma_{0}\left(s_{3}^{2}\right) \\
\Gamma_{0}\left(s_{2}^{3}\right)=-1\end{array}$ & $\mathbb{Z}_{2}$ & 3 & $21-d$ & $\bigoplus_{i=0}^{2} \Gamma_{i}$ & $\begin{array}{l}2 \text { identical: } \\
\text { Fermi, Bose }\end{array}$ \\
\hline (e) & $\begin{array}{l}\Gamma_{0}\left(s_{1}^{2}\right) \\
\Gamma_{0}\left(s_{1}^{3}\right)=-1\end{array}$ & $\mathbb{Z}_{2}$ & 3 & $21-\mathrm{d}$ & $\bigoplus_{i=0}^{2} \Gamma_{i}$ & $\begin{array}{l}2 \text { identical: } \\
\text { Fermi, Bose }\end{array}$ \\
\hline (f) & $\begin{array}{l}\Gamma_{0}\left(s_{1}^{2}\right) \\
\Gamma_{0}\left(s_{3}^{2}\right)=-1\end{array}$ & $\mathbb{Z}_{2}$ & 3 & $21-\mathrm{d}$ & $\bigoplus_{i=0}^{2} \Gamma_{i}$ & $\begin{array}{l}2 \text { identical: } \\
\text { Fermi, Bose }\end{array}$ \\
\hline (g) & $\begin{array}{l}\Gamma_{0}\left(s_{1}^{2}\right) \\
\Gamma_{0}\left(s_{1}^{3}\right) \\
\Gamma_{0}\left(s_{2}^{1}\right)=-1\end{array}$ & $\{e\}$ & 6 & trivial & $\bigoplus_{i=0}^{5} \Gamma_{i}$ & all distinct \\
\hline (h) & $\begin{array}{l}\Gamma_{0}\left(s_{1}^{2}\right) \\
\Gamma_{0}\left(s_{3}^{2}\right) \\
\Gamma_{0}\left(s_{3}^{1}\right)=-1\end{array}$ & $\{e\}$ & 6 & trivial & $\bigoplus_{i=0}^{5} \Gamma_{i}$ & all distinct \\
\hline (i) & $\begin{array}{l}\Gamma_{0}\left(s_{1}^{2}\right) \\
\Gamma_{0}\left(s_{2}^{1}\right) \\
\Gamma_{0}\left(s_{3}{ }^{1}\right)=-1\end{array}$ & $\{e\}$ & 6 & trivial & $\bigoplus_{i=0}^{5} \Gamma_{i}$ & all distinct \\
\hline$(\mathrm{j})$ & $\begin{array}{l}\Gamma_{0}\left(s_{1}^{2}\right) \\
\Gamma_{0}\left(s_{2}^{3}\right) \\
\Gamma_{0}\left(s_{3}^{1}\right)=-1\end{array}$ & $\mathbb{Z}_{3}$ & 2 & $31-d$ & $\bigoplus_{i=0}^{1} \Gamma_{i}$ & $\begin{array}{l}\text { "cyclically identical": } \\
3 \text { types }\end{array}$ \\
\hline
\end{tabular}


It is relatively easy to see from this example that as the number of $\mathbb{R} P^{3}$ primes increases, there will be an increasing number of sectors in which the statistics is dictated by non permutation subgroups of $S_{N}$. For example, it is readily seen that any cyclic subgroup of $S_{N}$ can play this role. In fact, given that every finite group is isomorphic to a subgroup of a permutation group, it seems plausible that statistics can be determined by an arbitrary finite group, given a large enough number of $\mathbb{R} P^{3}$ primes.

\section{$\S 7$. Conclusions}

Our main goal in this paper has been to understand physically the various conceivable quantum sectors (" $\theta$-sectors") which arise in 4 -dimensional quantum gravity when the spatial topology is non-trivial, but also non-dynamical. Mathematically, the task of finding all the quantum sectors translates (if we ignore the possibility of "Wess-Zumino terms" or other topological contributions to the quantum amplitude which modify the classical limit of the theory) into the problem of classifying the UIR's of the mapping class group $G$ of the spatial 3-manifold $\mathcal{M}$. Ideally one would wish for a full classification, but that is out of reach at present, if only because the prime 3-manifolds themselves remain unclassified. In the present paper we have concentrated on bringing out the specific features which arise because more than one geon is present.

In general $\mathcal{M}$ will be a connected sum of prime manifolds, and we have based our physical interpretation on a picture which regards each such prime (excluding the handles) as giving rise, on quantization, to a physical particle or topological geon. Our analysis has aimed at characterizing the UIR's of $G$ in the particle language appropriate to such a geon interpretation.

In one important case, our analysis has been complete in the sense that we have classified all possible multi-geon UIR's in terms of the UIR's of the mapping class groups, $G_{i}$, of the individual primes. The case we are referring to is the one in which the slidediffeomorphisms are represented trivially, so that $G$ reduces in effect to the "particle subgroup" $\widetilde{G}$ generated by the internal diffeomorphisms of the individual primes and the permutations of identical primes among themselves. Our classification of the UIR's of $\widetilde{G}$ was based on the decomposition of $\widetilde{G}$ as a semidirect product of the internal diffeomorphisms $G_{\text {int }}$ with the permutations $S_{N}$. From this decomposition there followed a characterization of the UIR's of $G$ in terms of the UIR's of the $G_{i}$ on one hand, and the 
UIR's of certain subgroups ("little groups") of the permutations on the other hand. (The mathematical facts used in obtaining the classification were summarized in $\S 3$ above.)

The characterization of the UIR's of $G$ in this manner lends itself to a mode of expression in which the "internal" UIR's (i.e. the UIR's of the internal diffeo groups $G_{i}$ ) determine the physical identity of the corresponding geons (their "quantum numbers" or "species"), while the UIR of the resulting little subgroup of $S_{N}$ determines the statistics of the geons. In this language, the classification scheme of $\S 4$ states that one obtains the general UIR of $\widetilde{G}$ by $(i)$ choosing a geon species for each prime which is present (i.e. a UIR of its MCG), and then ( $i i)$ choosing a statistics for each species of geon (i.e. a UIR of $S_{n}$, where $n$ is the number of geons of that species which are present). Notice that in general there will be more types of geons present than distinct diffeomorphism classes of primes, because a single type of prime can carry many different UIR's of its internal diffeomorphism group: "quantum breaking of indistinguishability". This mode of description of the UIR's seems fully satisfactory from a physical point of view, and its success in turn should offer strong support to the interpretation of primes as particles.

In the more general case of UIR's $R$ of $G$ for which the slides are not trivial, one may attempt a similar analysis based on the fact that the full mapping class group $G$ admits a (two-stage) decomposition into semidirect products. Once again, this analysis suggests definite criteria for when the geons should be called identical, and for what type of statistics the identical geons then exhibit. When slides are involved however, the harmony between the group theoretical analysis and the physical behavior of the geons is less perfect than for $\widetilde{G}$ alone, the problem deriving in some sense from the nonlocal character of the slides. Indeed we have sketched in $\S 6$ an alternative mode of description which would interpret geon identity and statistics somewhat differently, and it should be borne in mind that some of the detailed points we will make in the summary below would have to be stated differently if the alternative mode of description were adopted.

Aside from this purely interpretational difficulty resulting from the slides, a more serious problem is that the general form of the UIR's of the slide subgroup $\mathcal{S}$ is not known, and hence a general account of the UIR's of $G$ cannot be given. However, one may still start with some UIR's $\Gamma_{0}$ of $\mathcal{S}$ that one does know (e.g., abelian UIR's) and use them to build up a family of UIR's of $G$. In this manner, a large number of "theta sectors" can be found, and some of them introduce new effects, including previously unknown possibilities for particle identity and statistics, as described earlier in $\S \S 5$ and 6 . 
By way of summary, we now list some of the general conclusions on geon identity, statistics, and "internal symmetry" which have emerged from the analysis of this paper.

\section{(a) Geon identity}

(i) Primes that are diffeomorphic, and therefore classically identical, will give rise to distinguishable geons if their internal groups are represented inequivalently. (For example, if $\mathcal{P}$ is a prime with $\mathcal{R}_{2 \pi} \neq e$ and if $\mathcal{M}=\mathbb{R} \# \mathcal{P} \# \mathcal{P}$, then representing one of the $\mathcal{R}_{2 \pi}$ 's by +1 and the other by -1 will result in two geons of opposite spin-type, one tensorial and the other spinorial.)

(ii) Even if two primes carry the same representation in the sense of (i), it is possible for the slides to render them distinguishable. Examining the internal structures of individual geons is therefore inadequate to determine whether they are truly identical; one must study geon-geon collisions as well.

\section{(b) Geon Statistics}

(i) There will always exist sectors in which the spin-statistics correlation for geons is violated. Indeed, this occurs for an arbitrary prime $\mathcal{P}$ and an arbitrary geon type based on $\mathcal{P}$ (i.e. an arbitrary UIR of the MCG of $\mathcal{P} \# \mathbb{R}^{3}$ ). In particular, the geons can be arranged to be all bosonic or all fermionic irrespective of whether they are tensorial or spinorial. (How the internal diffeomorphism group of a prime is represented determines whether the corresponding geon is spinorial or tensorial.)

(ii) When $N \geq 3$ there will always exist sectors in which the geons obey parastatistics.

(iii) In the case of $\mathbb{R} P^{3}$ geons, when $N \geq 3$ there will always exist sectors in which geon identity and statistics are expressed not by a permutation subgroup of $S_{N}$ but by (for example) a cyclic subgroup of $S_{N}$. (We constructed such sectors in $\S 6$ using certain abelian representations of the slide subgroup). This type of behavior seems to be new. Moreover, Balachandran has pointed out that the same thing can in principle occur for string-like excitations in suitable condensed matter systems [22].

(iv) For $N \geq 4$ it may be that there also exist sectors in which statistics is expressed by a projective representation of $S_{N}$ or its subgroups, rather than an ordinary representation.

(v) For a pair of $\mathbb{R} P^{3}$ geons, there exist quantum sectors for which the bose-fermi distinction becomes ambiguous: one has pairs of sectors differing only by the sign 
of the exchange, without being able to say which sector is the bosonic one and which the fermionic.

\section{(c) Internal Symmetry of Geons}

(i) There exist quantum sectors for certain three manifolds in which the slides render the geons internally asymmetric even though classically the primes possess symmetries (expressed by their internal diffeo groups). Thus the examination of an isolated prime $\mathcal{P}$ is not adequate to determine its quantum symmetries; one must look at geon-geon collisions as well.

The existence of such novel aspects of geon behavior as listed above bespeaks a richness of quantum gravity deriving from the possibility of non-Euclidean topology inherent in general relativity. In itself, this novelty is intriguing, but not at all disturbing. However, what would appear to be disturbing is the huge degree of ambiguity in quantization associated with all these different UIR's of $G$. For example, even in the very simplest case where $\mathcal{M}=\mathbb{R}^{3} \# \mathbb{R} P^{3} \# \mathbb{R} P^{3}$, we saw that there arise an infinite number of two dimensional sectors parameterized by a real number $\tau$. Within the particle picture this implies an infinite number of possible geon-types for nature to choose from (even though the spatial topology is fixed). And with more (and more generic) primes present, one would expect far more free parameters, if not a discrete infinity of possible sectors. ${ }^{*}$ This does not seem to accord with the conception of quantum gravity as a fundamental theory. (Indeed, even classically, it seems a mystery how nature could have chosen one out of the discrete infinity of possible topologies for $\mathcal{M}$.)

[ One might object at this point that perhaps our last complaint is not well founded because not all these sectors are really possible dynamically. Indeed, our analysis so far has been on a purely "kinematical" level, in the sense that, basically, we have used nothing more than the facts that general relativity is a generally covariant theory possessed of a metric field $g_{a b}$. We have not used the specific dynamics of the Einstein-Hilbert Lagrangian (manifested in a canonical setting by the Hamiltonian constraints), so how do we know that this dynamics will not exclude most of the UIR's we have been examining? In view of this possible objection, we will pause for a moment and try to make it plausible that in

* It is not clear whether the free parameters which arise in this manner from spatial topology are related to the free parameters proposed to arise in connection with certain types of topological fluctuations ("wormholes") 23]. 
fact no sector is excluded by the dynamics. For definiteness we conduct the discussion in the language of canonical quantization rather than that of the path-integral.

[ According to reference [24], there is in 3-dimensions no topological obstruction to the specification of asymptotically flat initial data. Thus for each $\mathcal{M}$ we have considered, there exists at least one classical $3-$ metric ${ }^{3} g_{a b}$ which (together with an appropriate extrinsic curvature) solves the classical initial value constraints. Now classical general relativity is by definition the classical limit of quantum gravity; moreover we may scale our classical initial data to be as far as desired from the Planck scale. Hence we may reasonably assume the existence in the quantum theory of a WKB wavefunction $\psi$ peaked at a single 3 -metric ${ }^{3} g$, or rather on the set of $\left[{ }^{3} g\right]$ 's obtained by the different allowed slicings of the classical spacetime corresponding to our initial data, where $\left[{ }^{3} g\right]$ denotes here the set of metrics related to ${ }^{3} g$ by small diffeomorphisms. (Recall that invariance under the small diffeomorphism group $\operatorname{Diff}_{0}^{\infty}(\mathcal{M})$ suffices to satisfy the momentum constraints.)

[ Under the action of a nontrivial element of the mapping class group $G$, the metric ${ }^{3} g$ goes into a gauge-related metric ${ }^{3} g^{\prime}$, which, by definition belongs to a different equivalence class from that of ${ }^{3} g$, i.e. $\left[{ }^{3} g\right] \neq\left[{ }^{3} g^{\prime}\right]$. Thus, $G$ takes the equivalence class $\left[{ }^{3} \mathrm{~g}\right]$ into an arbitrary element of the fiber of $\mathcal{R} / \operatorname{Diff}_{0}^{\infty}$ which lies over the 3-geometry $q=\operatorname{cls}\left({ }^{3} g\right) \in Q=\mathcal{R} / \operatorname{Diff}^{\infty}$. The fact that the images of $\left[{ }^{3} g\right]$ by the elements of $G$ are all distinct elements of the fiber (remember that the action of Diff ${ }^{\infty}$ is free) makes it plausible that the images of $\psi$ by $G$ are all linearly independent. (In general a given 3-geometry will occur only once among the slices of 4-metric. Note also, that we don't really need independence of all the images when this number is infinite; if we only want to reproduce a finite dimensional UIR, it will suffice to choose ${ }^{3} g$ "macroscopic enough" so that we have a sufficiently large finite independent set.)

[ The set of WKB wavefunctions $\left\{\psi_{i}\right\}$ obtained in this way spans a vector subspace $\mathcal{H}$ of the full Hilbert space which naturally carries the regular representation of $G$ (or some sufficiently large portion thereof). Hence any UIR will be realizable in $\mathcal{H}$, whence in the state space of our theory. (Since $\psi$ satisfies all the constraints, so will the elements of $\mathcal{H}$.) By this reasoning, our analysis of the possible quantum sectors should be independent of the particular dynamics imposed, as long as that dynamics allows arbitrary topologies to occur in the classical limit. ]

As we have alluded to more than once, the plethora of quantum sectors described in this paper arises in theories of quantum gravity in which the spatial topology is "frozen". 
A natural question to ask is whether "thawing" the topology would remove the redundant sectors, among which the most plainly unphysical are those which violate the spin-statistics correlation. The most obvious framework in which the spacetime topology can fluctuate is the sum-over-histories (cf. [25]), and a set of rules for regaining the spin-statistics correlation in such a framework has been suggested in [2]. In [26] it has been shown that, even without imposing any new conditions like those of [2], the sum-over-histories automatically reproduces the spin-statistics correlation for certain pairs of identical geons (lens spaces) formed via a certain type of "U-tube cobordism". Other examples where topology change excludes sectors of the frozen theory have been given in [27] and [28].

The existence of redundant quantum sectors is not limited to quantum gravity. An example from molecular physics is the "rigid Born-Oppenheimer approximation". There the electronic degrees of freedom and the fluctuations in the shape of the molecule have been "frozen", and one is left with a rigid 3-dimensional body. When the shape of the body is symmetrical, the resulting configuration space is multiply connected, and this leads to inequivalent quantum sectors in the familiar manner (including sectors in which a kind of "quantum chirality" or "parity breaking" occurs [29]). However, not all of these sectors are truly possible for a given molecule. Rather the rigid body is only an effective description of the molecule. When one restores the degrees of freedom underlying this effective description (its "material base"), one finds [30 that some of the sectors are removed and the others brought together into a unified state space (unified because the strict superselection rule separating the sectors is lifted.)

We may take this as a typical pattern and a clue to what is happening in quantum gravity. On this view, incorporating topology change should restore some of the missing degrees of freedom and remove some of the redundancy. We suspect however that topological fluctuations alone will not suffice to remove the infinity of quantum sectors, and that the remaining redundancies will disappear only in a deeper theory based on a discrete structure like that of the causal set [31].

\section{Acknowledgments}

We would like to thank Fay Dowker for several discussions. We also would like to thank A.P. Balachandran for reading the first draft of this paper and for the many discussions that followed. Finally, we are grateful to Nico Giulini for a key suggestion in connection with the Appendix, and for providing us with related references. This 
research was partly supported by NSF grant PHY-9600620 and by a grant from the Office of Research and Computing of Syracuse University.

\section{Appendix}

In the main text we described generators for the mapping class group $G$ which go by the names of "slides", "internal diffeos" and "exchanges", and we designated the three subgroups of $G$ which these generate by the respective symbols $\mathcal{S}, G_{i n t}$ and $S_{N}$. We also demonstrated, using developments, that $\mathcal{S}$ is a normal subgroup of $G$ and that $G_{\text {int }}$ is invariant under conjugation by elements of $S_{N}$. This suffices to establish the inclusions of normal subgroups, $\mathcal{S} \subseteq \mathcal{S} G_{i n t} \subseteq \mathcal{S} G_{i n t} S_{N}=G$ and $G_{i n t} \subseteq G_{i n t} S_{N}=\widetilde{G}$. If we knew in addition that $\left(\mathcal{S} G_{i n t}\right) / \mathcal{S}=G_{i n t}, G / \mathcal{S} G_{i n t}=S_{N}$, and $G / \mathcal{S}=\widetilde{G}$, then we would have established the semidirect product decomposition (2) of $\S 2$ on which we based most of our analysis. In this Appendix, assembling most of the necessary ingredients from the literature, we sketch a proof of the above equalities, or rather a proof of (2) itself, together with certain other assertions we made in $\S 2$. Specifically we demonstrate the following facts. (Our notation for semidirect product has been explained in $\S 2$.)

\section{Facts to be shown}

(0) $G=\mathcal{S} \ltimes G_{\text {int }} \ltimes S_{N}$

(1) $S_{N}$ is isomorphic to the permutation group on $N$ elements (as its name implies).

(2) $G_{\text {int }}=G_{1} \times G_{2} \times \cdots \times G_{N}$

(3) $\mathcal{S}$ is described correctly by the generators and relations given in Section 2.

Lemma Let $G=N K$ with $N$ normal in $G$, let $\bar{G}=\bar{N} \ltimes \bar{K}$, and let $f: G \rightarrow \bar{G}$ such that

(i) $f(N) \subseteq \bar{N}$ and $f(K) \subseteq \bar{K}$

(ii) either (a) $f \mid N$ is injective, or (b) $f \mid K$ is injective

(iii) $f$ is surjective.

Then $G=N \ltimes K$.

Moreover in case (a) $N \simeq \bar{N}$, and in case (b) $K \simeq \bar{K}$.

Proof: straightforward.

Henceforth we will assume the truth of the Poincaré conjecture. We need this, because we will rely on the standard device of replacing diffeomorphisms by their action 
on loops in $\mathcal{M}$, and such a device obviously would not succeed in connection with primes which have no nontrivial loops.

In the following we write $\pi$ for $\pi_{1}(\mathcal{M}), \Phi$ for the homomorphism of $G \operatorname{into} \operatorname{Aut}(\pi)$ which takes a diffeomorphism into its natural action on loops in $\mathcal{M}$, and $\bar{G}$ for $\Phi(G)$, the image of $G$ in $\operatorname{Aut}(\pi)$. Similarly, we write $\overline{\mathcal{S}}, \overline{G_{i n t}}$ and $\bar{S}_{N}$ for the images of these subgroups in $\operatorname{Aut}(\pi)$.

\section{Lemma on the structure of $\bar{G}$}

$$
\bar{G}=\overline{\mathcal{S}} \ltimes \bar{G}_{i n t} \ltimes \bar{S}_{N}
$$

If we use the generators described in the text, then this lemma follows directly from the structure of the Fuks-Rabinovitch (F-R) relations, as presented in [6] [17]. In fact all of the latter are relations within one of the three subgroups of the lemma, or else they specify how one of the subgroups acts on the generators of another. This, plus the obvious fact that the exchanges leave $\overline{G_{\text {int }}}$ invariant, is all we need to establish the lemma. (In order to be sure that the F-R relations are complete, we must know that the individual factors $\pi_{1}\left(\mathcal{P}_{j}\right)$ of $\pi$ are not further decomposable as free products. This is the content of "Kneser's Conjecture", Theorem 7.1 of [15].)

We take from [21] the following

Fact $\operatorname{ker}(\Phi) \subseteq G_{\text {int }}$.

(In comparing with that reference, notice that "rotations parallel to the connected sum 2-spheres" are included in what we call $G_{i n t}$; physically such a diffeomorphism represents a $2 \pi$ rotation of the corresponding geon.) Further, we have,

Claim $1 \Phi \mid S_{N}$ is injective

This follows trivially from fact that $\pi=\pi_{1}(\mathcal{M})$ is the free product of the individual $\pi_{1}\left(\mathcal{P}_{i}\right)$ (all of which are nontrivial, by the Poincaré conjecture): for any permutation $p$ we can find an element of one $\pi_{1}$ which goes to an element of a different $\pi_{1}$, and these cannot be equal in a free product.

Claim $2 \Phi \mid \mathcal{S}$ is injective

This follows from the fact that the generators of $\mathcal{S}$ (i.e. the slide diffeomorphisms described in Section 2) already satisfy all of the F-R relations that involve only the "slide generators" of $\bar{G}$, which in turn can be seen, for example, by using developments as in $\S 2$. 
Now let us apply our first lemma with $N=\mathcal{S}, K=G_{\text {int }} S_{N}, G=G$ and $f=\Phi$. We know that $N$ is normal in $G$, while $\bar{G}=\bar{N} \ltimes \bar{K}$ follows from our second lemma. It is trivial that $f$ is surjective and that $f(N) \subseteq \bar{N}, f(K) \subseteq \bar{K}$. Finally we have that $f \mid N$ is injective from Claim 2 above. Hence case (a) of the lemma applies and tells us both that

$$
G=\mathcal{S} \ltimes\left(G_{i n t} S_{N}\right)
$$

and that $\mathcal{S}=\overline{\mathcal{S}}$. The latter is item (3) in our list of facts to be proven, because the commutation relations quoted in $\S 2$ are a complete set of relations for $\overline{\mathcal{S}}$ according to [6] [17].

Now let us apply our first lemma again with $N=G_{\text {int }}, K=S_{N}$ and with $G$ being the "particle group", $G_{i n t} S_{N}=\widetilde{G}$. By Claim 1 above, we are in case (b) of the lemma , so we conclude

$$
\widetilde{G}=G_{i n t} \ltimes S_{N}
$$

and also $S_{N}=\bar{S}_{N}$. The latter establishes item (1) of our list, since the generators and relations of $\bar{S}_{N}$ are precisely those of the permutation group on $N$ elements.

Putting the last two results together yields $G=\mathcal{S} \ltimes\left(G_{\text {int }} \ltimes S_{N}\right)$, which is equivalent to item (0) in our list of facts to be proven.

It remains to demonstrate item (2), or in other words equation (1) of the main text. As mentioned in $\S 2$, this isomorphism almost follows trivially from the fact that the each $G_{i}$ can be defined with support on its own prime, but because the supports need not be disjoint, we strictly speaking have not yet excluded that some combination of elements of different $G_{i}$ 's might vanish. In that case, equation (1) would not be an equality, but only a surjective map $G_{1} \times G_{2} \times \cdots \times G_{N} \rightarrow G_{\text {int }}$.

The needed proof that this map is in fact injective has been given in [32], using the following argument. According to theorem 1 of [20], Diff ${ }^{\infty}(\mathcal{M})$ is a trivial principal fibre bundle with fibre $D_{1} \times D_{2} \times \cdots D_{N}$, where $D_{i}=\operatorname{Diff}^{\infty}\left(\mathbb{R}^{3} \# \mathcal{P}_{i}\right)$ is the group of internal diffeomorphisms of the $i^{t h}$ prime. The base space $\operatorname{Imb}$ of this bundle is a space of embeddings of $B$ into $\mathcal{M}$, where $B$ is the submanifold of $\mathcal{M}$ that remains when the primes are "cut out" of $\mathcal{M}$. (Specifically, an embedding belongs to Imb iff it extends to an element of $\operatorname{Diff}^{\infty}(\mathcal{M})$.) Thus we have the homeomorphism,

$$
\operatorname{Diff}^{\infty}(\mathcal{M}) \simeq D_{1} \times D_{2} \times \cdots \times D_{N} \times \operatorname{Imb}
$$


Since in general $\pi_{0}(A \times B)=\pi_{0}(A) \times \pi_{0}(B)$, this implies that the fibre inclusion,

$$
D_{1} \times D_{2} \times \cdots \times D_{N} \rightarrow \operatorname{Diff}^{\infty}(\mathcal{M})
$$

induces an injective homomorphism

$$
\begin{aligned}
G_{1} \times G_{2} \times \cdots \times G_{N} & =\pi_{0}\left(D_{1}\right) \times \pi_{0}\left(D_{2}\right) \times \cdots \times \pi_{0}\left(D_{N}\right) \\
& =\pi_{0}\left(D_{1} \times D_{2} \times \cdots \times D_{N}\right) \rightarrow \pi_{0}\left(\operatorname{Diff}^{\infty}(\mathcal{M})\right)=G .
\end{aligned}
$$

Moreover, the image of $G_{1} \times G_{2} \times \cdots \times G_{N}$ by this mapping is by definition the internal group $G_{i n t}$, which therefore is isomorphic to $G_{1} \times G_{2} \times \cdots \times G_{N}$, as claimed. 


\section{References}

[1] A.P. Balachandran, "Classical Topology and Quantum Phases: Quantum Mechanics" in Filippo, S. de, Marinaro, M., Marmo, G., (eds.), Geometrical and Algebraic Aspects of Nonlinear Field Theories (Proceedings of the conference of the same name, held Amalfi, Italy, May 1988), 1-28, (Elsevier, Amsterdam, 1989)

[2] R.D. Sorkin, "Classical Topology and Quantum Phases: Quantum Geons", in Filippo, S. de, Marinaro, M., Marmo, G., (eds.), Geometrical and Algebraic Aspects of Nonlinear Field Theories (Proceedings of the conference of the same name, held Amalfi, Italy, May 1988), 201-218, (Elsevier, Amsterdam, 1989)

[3] R.D. Sorkin, "Introduction to Topological Geons", in P.G. Bergmann and V. de Sabbata (eds.), Topological Properties and Global Structure of Space-Time, (Proceedings of the conference of the same name, held Erice, Italy, May 1985), 249-270, (Plenum Press, 1986)

[4] C.J. Isham, "Topological and Global Aspects of Quantum Theory", in B.S. DeWitt and R.Stora (eds.), Relativité, Groupes et Topologie II", (Proceedings of the conference of the same name, held LesHouches, France, 1983), 1059-1290, (North-Holland, 1984)

[5] Ch. Aneziris, A.P. Balachandran, M. Bourdeau, S. Jo, T.R. Ramadas and R.D. Sorkin, "Aspects of Spin and Statistics in Generally Covariant Theories", Int. J. Mod. Phys. A4: 5459-5510 (1989)

[6] D.I. Fuks-Rabinovich, "On the Automorphism Groups of Free Products I" ("O gruppakh avtomorfismov svobodnykh proizvedenii. I") Mat. Sbornik, 8(50): 265-276 (1940); D.I. Fuks-Rabinovich, "On the Automorphism Groups of Free Products II", ("O gruppakh avtomorfismov svobodnykh proizvedenii. II") Mat. Sbornik, 9(51): 183-220 (1941)

[7] G.W. Mackey, "Induced Representations of Groups and Quantum Mechanics", (W.A. Benjamin Inc. and Editore Boringhieri, 1968); G.W. Mackey, "Theory of Unitary Group Representations", (University of Chicago Press, 1976); G.W. Mackey, "Unitary Group Representations in Physics, Probability and Number Theory", Addison-Wesley Publishing Co. Inc., 1978

[8] A.H. Clifford, "Representations Induced in an Invariant Subgroup", Annals of Mathematics 38: 533-550 (1937)

[9] Sumati Surya, Ph.D. Thesis (in preparation)

[10] J.L. Friedman and R.D. Sorkin, "Spin-1/2 from Gravity", Phys. Rev. Lett. 44: 11001103 (1980); and 45:148 (1980) J.L. Friedman and R.D. Sorkin, "Half-Integral Spin from Quantum Gravity", Gen. Rel. Grav. 14:615-620 (1982) 
[11] R.D. Sorkin, "On the Role of Time in the Sum-over-histories Framework for Gravity", paper presented to the conference on The History of Modern Gauge Theories, held Logan, Utah, July, 1987; published in Int. J. Theor. Phys. 33:523-534 (1994)

[12] R.D. Sorkin, "Quantum Measure Theory and its Interpretation", in D.H. Feng and B-L Hu (eds.), Proceedings of the Fourth Drexel Symposium on Quantum Nonintegrability: Quantum Classical Correspondence, held Philadelphia, September 8-11, 1994 (World Scientific, in press) <e-Print Archive: gr-qc/9507057>

[13] Michael G.C. Laidlaw and Cécile Morette DeWitt, "Feynman Functional Integrals for Systems of Indistinguishable Particles", Phys. Rev. D, 3: 1375-1378 (1971)

[14] H. Hendriks, “ Applications De La Théorie D’Obstruction En Dimension 3", Bull. Soc. Math. France, Memoire,53: 81 (1977). See Section 4.3.

[15] J. Hempel, "3 - Manifolds", Annals of Math. Study, No. 86, (Princeton University Press, Princeton, 1976)

[16] J.L. Friedman and D.M. Witt, "Internal Symmetry Groups of Quantum Geons", Phys. Lett. 120B: 324-328 (1983) D.M. Witt, "Symmetry Groups of State Vectors in Canonical Quantum Gravity", J. Math. Phys. 27: 573-592 (1986)

[17] D. McCullough and A. Miller, "Homeomorphisms of 3-Manifolds with Compressible Boundary" Memoires of the American Math. Soc., Vol. 61, No. 344 (1986) (See Chapter 5)

[18] D. Giulini, "Properties of Three Manifolds for Relativists", Freiberg-THEP-93-15 $<$ e-print archive: gr-qc/9308008>

[19] S.M. Coxeter and W.O.J. Moser, Generators and Relations for Discrete Groups, $4^{\text {th }}$ edition (Springer-Verlag, 1980)

[20] H. Hendriks and D. McCullough, "On the Diffeomorphism Group of a Reducible Three Manifold", Topology Appl., 26: 25-31 (1987)

[21] D. McCullough, "Topological and Algebraic Automorphisms of Three Manifolds", in R.A. Piccinini (ed.), Groups of Self Equivalences and Related Topics, 102-113, (Springer Lecture Notes in Mathematics, 1425) (Springer Verlag, 1988)

[22] A.P. Balachandran (private communication)

[23] S.W. Hawking and R. Laflamme, "Baby Universes and the Nonrenormalizability of Gravity" Phys. Lett. 209B : 39 (1988); Sidney Coleman, "Black Holes as Red Herrings", Nuclear Physics B 307: 867-882 (1988); Steven B. Giddings and Andrew Strominger, "Loss of Incoherence and Determination of Coupling Constants in Quantum Gravity", Nuclear Physics B 307: 854-866 (1988)

[24] Donald M. Witt, "Vacuum Space-Times That Admit No Maximal Slice", Phys. Rev. Lett. 57 : 1386-1389, (1986) 
[25] Arvind Borde and Rafael D. Sorkin, "Causal Cobordism: Topology Change Without Causal Anomalies", (in preparation)

[26] H.F. Dowker and R.D. Sorkin, "A Spin Statistics Theorem for Certain Topological Geons", <e-print archive: gr-qc/9609064>

[27] J.B. Hartle and D.M. Witt, "Gravitational Theta States and the Wavefunction of the Universe", Phys. Rev. D, 37: 2833-2837 (1988)

[28] Domenico Giulini and Jorma Louko, "No Boundary Theta Sectors in Spatially Flat Quantum Cosmology", Phys. Rev. D, 37: 4355-4364 (1992)

[29] A.P. Balachandran, A. Simoni and D.M. Witt, "Molecules and how they violate P and T", Int. J. Modern Phys. A7:2087-2107 (1992)

[30] A.P. Balachandran and S.M. Vaidya, "Parity Doubles in Quark Physics", Phys. Rev. Lett. 78:13-16 (1997) <e-Print Archive: hep-ph/9606283>; "Emergent Chiral Symmetry: Parity and Time Reversal Doubles" (Syracuse University preprint SU-4240-653) $<$ e-print archive: hep-th/9612053>

[31] L. Bombelli, J. Lee, D. Meyer and R.D. Sorkin, "Spacetime as a causal set", Phys. Rev. Lett. 59:521 (1987); L. Bombelli, Space-time as a Causal Set, Ph.D. dissertation (Syracuse University, 1987); D.A. Meyer, The Dimension of Causal Sets, Ph.D. dissertation ( M.I.T., 1988); G. Brightwell and R. Gregory, "The Structure of Random Discrete Spacetime", Phys. Rev. Lett. 66:260-263 (1991); A.R. Daughton, "The Recovery of Locality for Causal Sets and Related Topics", Ph.D. dissertation (Syracuse University, 1993); J. Myrheim, "Statistical geometry," CERN preprint TH-2538 (1978); G. 't Hooft, "Quantum gravity: a fundamental problem and some radical ideas,", in M. Levy, S. Deser (eds.), Recent Developments in Gravitation (Proceedings of the 1978 Cargese Summer Institute), (Plenum, 1979)

[32] Domenico Giulini, "On the Configuration Space Topology in General Relativity", Helv. Phys. Acta, 68: 86-111 (1995), see pp. 91-92. 